\title{
Normal Stress Differences and Yield Stresses in Attractive Particle Networks
}

\author{
David I. Verrelli ${ }^{1}$ and Adam R. Kilcullen ${ }^{2}$ \\ ${ }^{1}$ Department of Biomedical Sciences, Macquarie University, North Ryde, NSW 2109, Australia \\ ${ }^{2}$ Department of Chemical and Biomolecular Engineering, The University of Melbourne, Parkville, VIC 3010, Australia \\ Correspondence should be addressed to David I. Verrelli; david.verrelli@mq.edu.au
}

Received 13 August 2015; Accepted 1 December 2015

Academic Editor: Shengqiang Cai

Copyright (C) 2016 D. I. Verrelli and A. R. Kilcullen. This is an open access article distributed under the Creative Commons Attribution License, which permits unrestricted use, distribution, and reproduction in any medium, provided the original work is properly cited.

\begin{abstract}
The nature of attractive particulate networks, yield stresses, and normal stress differences is systematically reviewed, each in terms of the relevant definitions, underlying mechanisms, and current measurement techniques. With this foundation, experimental observations of normal stress differences in some suspensions and colloidal systems are surveyed, along with constitutive models that allow for normal stress differences to arise prior to yielding. Yield stresses are a hallmark of attractive colloidal systems and vital in their processing. In contrast, little attention has been given to the role of normal stress differences in these systems. The presence or absence of normal stress differences necessarily affects the isotropy of the normal stress field through the solid particulate phase (treated as a continuum), in turn affecting estimation of yield stress. Given the importance of yield stresses in dealing with practical industrial problems, and in understanding fundamental behaviours, it is important to ensure that yield measurements can be relied upon.
\end{abstract}

\section{Introduction}

The literature abounds with studies of either normal stress differences $\left(N_{1}\right.$ and $\left.N_{2}\right)$ or yield stresses $\left(\tau_{\mathrm{y}}\right)$, but seldom are both discussed for a given material. Most of the studies on normal stress differences are conducted on polymeric systems (i.e., melts and solutions), where these phenomena are large in magnitude, while yield stresses are negligible or nonexistent. Yield stresses are manifest as obvious features in a range of other systems, in which interaction between "solid" components is a characteristic; in the present discussion we will focus on colloidal particulate systems, in which the particles are attracted to one another, or "cohesive."

The practical absence of yield stresses in polymeric systems does not correspond to an absence of normal stress differences in attractive colloidal systems. Indeed, evidence from direct experimental observation, indirect experimental inference, and predictions from mathematical models all suggest that colloidal particulate systems can exhibit significant normal stress differences.
When a given sample exhibits both a yield stress and normal stress differences, we would like to know whether the two phenomena operate in isolation, or whether one can influence the other. This will be addressed by considering the physics involved and by comparing the magnitudes of the stresses involved.

The following discussion will focus on shear rheology. However, the principles can be applied to compressive rheology and extensional rheology, in which shear stresses are still to be found.

\section{Principles}

\subsection{Attractive Particle Networks}

2.1.1. Definitions. Attractive particle networks are often referred to as "gels," although their properties are different from polymer gels. According to Mewis and Wagner [1] there is "no single definition" of a gelled state. For our purposes, there are only two definitions that matter [2]: first, 
the primary particles are "bonded" to each other so as to span the volume; second, the solid phase can support a load. By "bonded" it is meant that individual solid components are associated with an attractive interaction-a slight surface separation is allowed (e.g., [3-5]). The two definitions are often conflated but strictly should be treated separately. Specifically, a network could form through floppy bonds that do not support any load, whereas a stable suspension at high concentration (i.e., a particulate "glass") may support a load without ever being "bonded." The first definition is the more fundamental, but it cannot readily be measured, and so recourse is usually made to the second definition.

2.1.2. Mechanism. Attractive particle networks are formed when two conditions are met: (i) the attractive forces between particles are sufficiently strong to withstand whatever other forces are acting, such as gravity or shear; (ii) the volume fraction of solids, $\varphi$ (i.e., solidosity), is high enough for the resulting interconnected set of particles to span the volume of the sample. If the attractions are too weak, or the solid volume fraction is too small, then the particles will only be able to form isolated clusters. An exhaustive review is beyond the scope of the present work; standard references include the books by Shaw [6], Hunter [7], Israelachvili [8], and Russel et al. [9].

Particulate networks found in industry and in nature are generally mixtures of diverse particle shapes, sizes, and (sometimes) compositions. The interparticle bonding in such systems can be caused by suppression of electrostatic repulsion so that van der Waals forces dominate (as in coagulation) $[6,7,10,11]$, or they can be due to polymer flocculation, which might be due to the formation of polymer bridges [7, 12, 13], or due to creation of charge patches on the particle surfaces due to adsorbed polymer $[14,15]$. Particle aggregation in a secondary minimum of the interaction potential can also occur (see $[7,12]$ ) but is not very important in practical systems [1].

Among a few other mechanisms of particle aggregation, one more that must be noted here is depletion flocculation. This involves addition of a nonadsorbing polymer to the fluid. The polymer is unable to access the interstices between nearby particles, and the osmotic force so generated favours reduction of interparticle gaps. This mode of aggregation is popular in rheological studies because the attraction can be "tuned" [16], and the aggregation is more-or-less reversible [12]. However, treatment in general texts is rather cursory (e.g., $[6,8]$ ), suggesting it is unimportant in most practical applications-perhaps due to the relatively weak attractions that result [8]. Depletion forces tend also to be relatively short-ranged [8], which engenders specific behaviours that do not occur with longer-ranged interactions [1].

At very low particle concentrations a network is eventually built up by the interconnection of aggregates or "flocs" that were formed by either "particle-cluster aggregation" or "cluster-cluster aggregation" (e.g., [17-19]); in contrast, at very high particle concentrations, a network forms in a statistically uniform way throughout the entire volume by "percolation" or other such processes (e.g., [20, 21]). Although the terms aggregation and percolation are occasionally used interchangeably in the literature, the processes are distinct and produce different microstructures [22-24].

Biological Systems. Colloidal networks also form in some biological systems (e.g., [25]). Typically in biological systems it is the cells that would represent the colloidal "particles." Examples include human red blood cells (erythrocytes) (e.g., $[26,27])$; algae (e.g., [28, 29]); and bacteria (e.g., [25, 30-32]).

These biological systems introduce new complexities not seen in nonbiological systems (e.g., [25, 26, 30-32]), ${ }^{1}$ as discussed further in the Supplementary Material available online at http://dx.doi.org/10.1155/2016/1716598. Therefore it is expected to be more fruitful to first investigate abiologic systems to gain understanding of the fundamental mechanical principles and subsequently seek to judiciously apply these principles to biological systems.

Reversibility. Besides special cases such as depletion flocculation, aggregation is not generally reversible [12, 24, 33]. Coagulation by charge-neutralisation may be reversible in principle (cf. [34]), but practical coagulation (such as the sweep coagulation practiced in water treatment [24]) is essentially irreversible (cf. [35]). This irreversibility is seen when aggressively shearing firm, "solid-like" gels such as soil samples [36] or dewatered water treatment plant sludge [24], as they cannot recover their original networked state after being liquefied.

Even when the bonding is reversible, the structural arrangement within and between individual clusters of particles can be highly dependent upon the shear regime during aggregation (e.g., [22]) and the local particle concentration $[22,37]$. When a particulate gel is disrupted and reforms, if the conditions are not just right, then it is likely to have a different network structure, and thus different physical properties (e.g., [38]).

The ideal approaches to avoid disturbance of the sample are to either measure it in situ (e.g., [39]) or to generate the sample in situ within the rheometer (e.g., [40]).

2.1.3. Measurement. The topic of general measurements on colloidal gels is too broad to be succinctly reviewed within the present scope and depends greatly upon the application. With respect to rheological measurements, the two main areas of interest are shear rheology and compressive rheology. The shear rheology of these materials can be exceedingly complicated, as discussed in standard texts $[1,41,42]$. Measurements illuminating the shear rheology are discussed in the following sections. Compressive rheology $[24,43]$ is worthy of more investigation, dealing as it does with complementary yielding and flow phenomena, but is beyond the scope of the present review. Extensional rheology of these systems is generally not considered (cf. [42]).

In rheological investigations it is common to impose a high "preshear" to aggregated samples to "rejuvenate" them and thereby engender a consistent shear history (cf. [44, 45]). This is a good strategy for fundamental investigations that require "well-behaved" specimens but is not necessarily appropriate for more applied investigations of industrial materials. Ovarlez et al. [46] also warn against preshearing. 


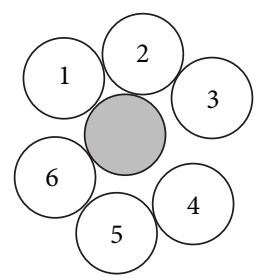

(a)

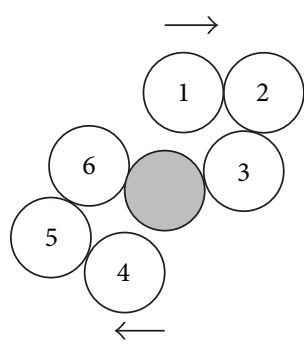

(b)

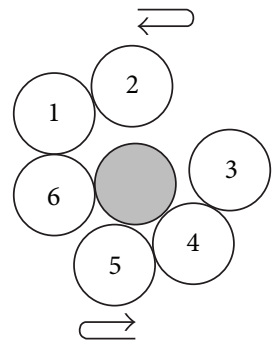

(c)

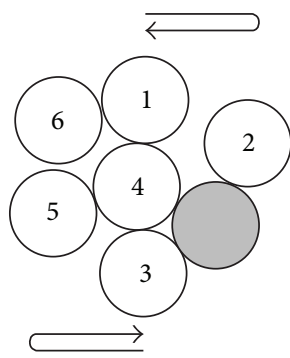

(d)

FIGURE 1: Yielding mechanisms in an attractive particulate glass, viewed along the vorticity direction. (a) The original unsheared configuration. The shaded particle has particles 1-6 as topological neighbours and is bonded to particles 1,2, and 6. Under small shear strains, the bonds are stretched but the particles stay in the same configuration. (b) Breakage of bonds above a threshold shear strain. (c) After one cycle of oscillatory shear, the shaded particle is now bonded to different particles. (d) Above another threshold in oscillatory shear strain, the identity of the topological neighbours around the shaded particle is changed and the glass is melted and becomes liquid-like. (Reprinted with permission from Pham et al. [59]. Copyright 2008, The Society of Rheology.) (See also [61, 156].)

An overarching feature of particulate gels is that the behaviour is not controlled purely by the solid phase but rather by a complex interplay of contributions from the particulate skeleton and the fluid in which the particles are embedded. Thus while the actual definition of a gel may not be so difficult, defining reliable characteristics that can be measured is more problematic.

\subsection{Shear Yield Stresses}

2.2.1. Definitions. The mathematical formulation of a yield stress can be traced back to Schwedoff, in 1900 [47]. ${ }^{2}$ Bingham [48] famously used the yield stress as a quantitative characterisation in preference to the less precise qualitative description of "plastic" flow that was conventional at the time. In Bingham's model a material would flow if exposed to shear stresses above a critical value, while it would act as a rigid solid under the action of smaller stresses. As measurement techniques became more sensitive, a debate arose as to whether yield stresses were "real," or whether the concept obscured the true behaviour [49-51]. The consensus established was that for most practical purposes it is reasonable to characterise material behaviour in terms of a yield stress, provided that the timescale and length scales of the measurement were commensurate with the relevant scales of the application [50, 52-57].

Associated with these developments, the concept of "yielding" has broadened to include not just the transition from bulk elastic solid to viscous-fluid behaviour, but also each of the mechanistic steps associated with breakdown of the particulate gel structure. A popular modern conception of particle networks holds that at long length scales the particles may cluster, but on short length scales they can be viewed as variants of hard-sphere glasses (cf. [58]) in which particles are kinetically "trapped" by "cages" formed of the neighbouring particles [59-61]. With reference to this view, the yielding steps are hypothesised to include a sequence of

(1) "bond breaking," or movement of the particles within their "cage";
(2) "cage breaking," or escape of the particle from the "cage";

(3) breaking of denser (stronger) clusters.

Prior to yielding, strain is due only to bond stretching (or rotation). The first two mechanisms are illustrated in Figure 1.

This conception has been built up largely through experiments on depletion flocculated spheres at relatively high solid volume fraction, $\varphi$ (e.g., $[59,60])$. At lower $\varphi$, it has been suggested that the interpretation should be adjusted, so that the first yielding event is associated with breakdown of bonds between clusters, while further yielding involves the breakdown (or densification) of these clusters [60]. We would support this latter view, especially in relation to strongly aggregated systems that can form particulate gels even at $\varphi \sim$ $0.001[62]$.

To be properly rigorous, the dependence of the shear yield stress, $\tau_{\mathrm{y}}$, upon isotropic ("hydrostatic") normal stresses should be considered, as should the existence of normal stress differences. This latter is especially important due to the common use, in studies of these materials, of the coordinate system suggested by the rheometric geometry (e.g., a parallel-plate or Couette cell), rather than principal stresses and strains as in solid mechanics [47, 63] (cf. [64, 65]). A correction for this is provided in Section 3.

Various shear yield strains associated with the respective shear yield stresses can also be defined $[1,59,66]$. The shear strain rate may also be important [67] (see Figure 3(b)).

2.2.2. Mechanism. Colloidal materials are able to act as an (elastic) solid while the constituent particles mutually attract, ${ }^{3}$ so as to form a particulate "network," "gel," or "paste." Only when sufficient stress is applied to overcome the interparticle forces can the bonds rupture and the network fail, such that the material begins to exhibit viscous (or viscoelastic) behaviour. Strongly aggregated systems tend to exhibit more clearly defined yield stresses, whereas weakly bonded particles are more likely to exhibit creep [24, 68-70] (cf. $[17,18,71]$ ). 
The yield stress arises from particle-particle bonds. The liquid phase does not (directly) contribute to this mechanism. The main roles of the liquid are to provide a degree of buoyancy to the solid phase and to provide an environment for counterions to modulate the overall interparticle forces. Nevertheless, the presence of liquid does superimpose a viscous damping effect on the response attributable to the particulate network alone [72]. In typical applications the liquid phase behaviour can be treated as Newtonian.

2.2.3. Measurement. There are numerous techniques for measuring shear yield stress, as described in various reviews $[1,24,42,53,57]$. These include the vane test $[49,73]$, the "slump test" $[74,75]$, penetration methods [39, 76-79], parallel-plate compressional "plastometry," "squeeze film" or "upsetting" devices $[49,79,80]$, incipient yielding on an inclined plane or channel [81, 82], capillary rheometers [49, $83]$, analytical rotational rheometers $[49,79,84]$, and triaxial testers [79]. Experimental comparisons of the shear yield stresses estimated using different techniques were presented by Yoshimura et al. [85] and James et al. [56].

Another (indirect) measure of yielding is commonly obtained by examination of the storage modulus, $G^{\prime}(\omega)$, and loss modulus, $G^{\prime \prime}(\omega)$, obtained from dynamic (oscillatory) tests at oscillation frequency $\omega$-at low strain amplitude (cf. [86]). It is often supposed that the point of equality of $G^{\prime}(\omega)$ and $G^{\prime \prime}(\omega)$ marks the transition between solid-like and liquid-like behaviour [41]. More rigorously, a critical gel condition is reached when both $G^{\prime}(\omega)$ and $G^{\prime \prime}(\omega)$ vary like $\omega^{n}$, in which $0.5 \leq n \leq 1$, corresponding to a (self-similar) power-law distribution of relaxation times (see [1]).

The cone-plate geometry remains much more popular in nonlinear dynamic tests, for procedural advantages including simplicity, smaller sample requirements, and familiarity. Yet this configuration is also susceptible to issues such as bridging of the small gap by particles in the sample (jamming) and slip effects.

The vane test (Figure 2) is quite straightforward and robust compared to the alternative techniques and so is discussed at greater length below.

Vane Techniques. The shear yield stress, $\tau_{\mathrm{y}}$, can usually (cf. [81]) be most reliably measured by inserting or embedding a "vane" $[49,73]$ into a sample and observing the stress, $\tau$, at a low, constant strain rate [53, 87-89]. An illustration of shear stress development as a function of time is presented in Figure 3(a). Usually the maximum shear stress, $\tau_{\mathrm{y} 3}$, is recorded as the "static" shear yield stress [53, 87-90]. This maximum stress has been proposed to correspond to the stress required to break the strongest bonds between the "kinetic units" of the system [55]. In the present context, "kinetic units" can be particle aggregates, single particles, or even particle fragments. ${ }^{4}$ Doraiswamy et al. [91] also warn that transition from solid-like to viscous behaviour is likely to occur over a range of shear strains for real materials. Coussot [72] further notes that gradual solid-liquid transitions may be observed due to (shear) stress heterogeneities. Formation of a peak "overshoot" in the shear stress, as in Figure 3(a), is also predicted in some constitutive models $[63,92]$. The breadth of

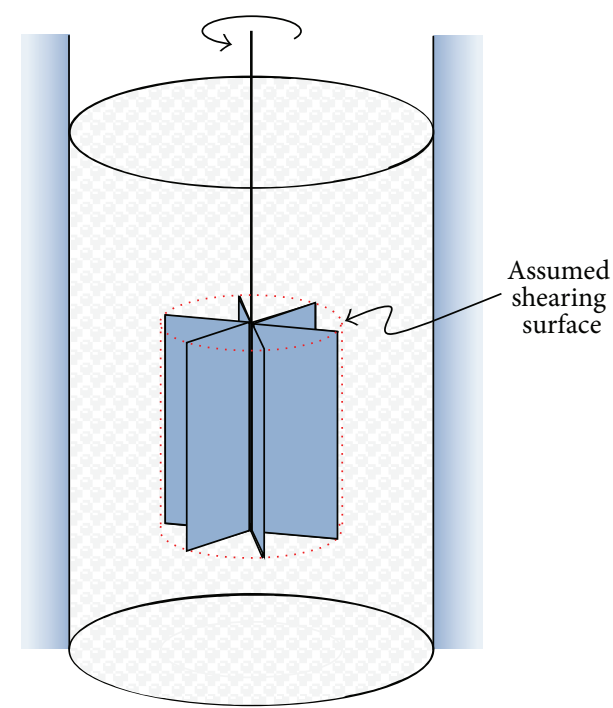

FIgURE 2: Schematic of one embodiment of a vane test. As per the dictum of Nguyen and Boger [90], the diameter and height of the sample are shown as (at least) double those of the vane here. The presumed shearing or slip "plane" is also sketched.

the peak is attributed to the stochastic nature of the breakup [59].

Notwithstanding the landmark paper of Nguyen and Boger [90] (cf. [73]), vane measurement techniques have been used for almost a century in soil mechanics, dating back to 1919 [39]. ${ }^{5}$ In soil mechanics recording the steady shear stress eventually attained after yielding is sometimes practiced $[89,93,94]$, which is $\tau_{\mathrm{y} 4}$ in Figure 3(a). This can be called a "dynamic" shear yield stress [54]. At this steady-state condition the rates of bond breakage and reformation are expected to become equal [95]. This stress can be compared with the "dynamic" shear yield stress obtained by lowering the shear strain rate, $\dot{\gamma}$, until the shear stress approaches a constant value $[41,56]$.

Another yield point can be defined as the shear stress at which the stress-time curve first deviates from linearity, marked as $\tau_{\mathrm{y} 1}$ in the sketch (cf. [55]). Given the application of a constant strain rate, this point indicates the end of the linear elastic region. Clearly the elastic behaviour observed at small strains is due to reversible deformation of particleparticle bonds. In a simplified analysis the end of the linear elastic region would be identified with the commencement of plastic deformation associated with the rupture of particleparticle bonds $[53,95]$. In fact, nonlinear elasticity may also occur $[54,96]$, so that rigorously we may only suppose that plastic yielding begins somewhere between $\tau_{\mathrm{y} 1}$ and $\tau_{\mathrm{y} 3}$, which is marked in the sketch as $\tau_{\mathrm{y} 2}$. (If linear plasticity were allowed too, then both $\tau_{\mathrm{y} 1}$ and $\tau_{\mathrm{y} 2}$ could shift to lower values. Although equations are sometimes expediently borrowed from the elasticity literature implying a linear plastic response $[93,97]$, we are not convinced of a physical basis for that.)

The "critical" shear strains $\gamma_{\mathrm{e} 1}$ and $\gamma_{\mathrm{e} 2}$, corresponding to $\tau_{\mathrm{y} 1}$ and $\tau_{\mathrm{y} 2}$, are the limits for linear deformation and elastic deformation, respectively. Such critical values may 


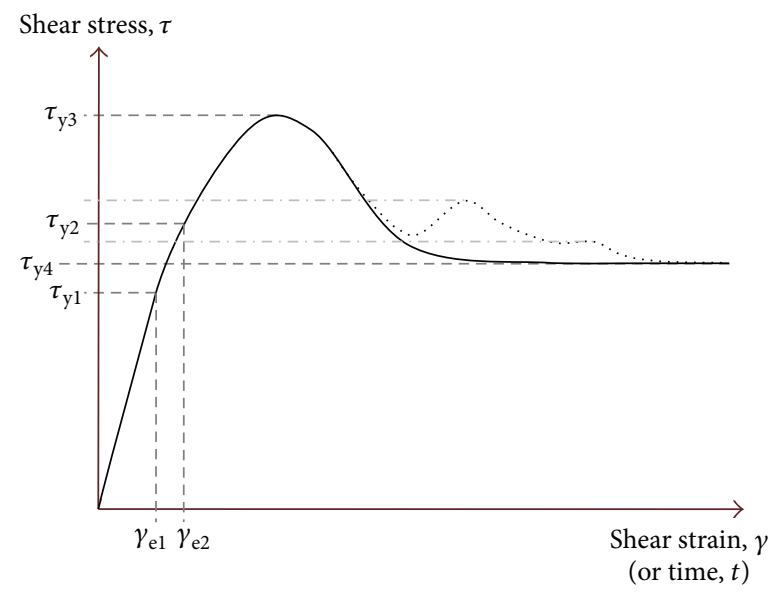

(a)

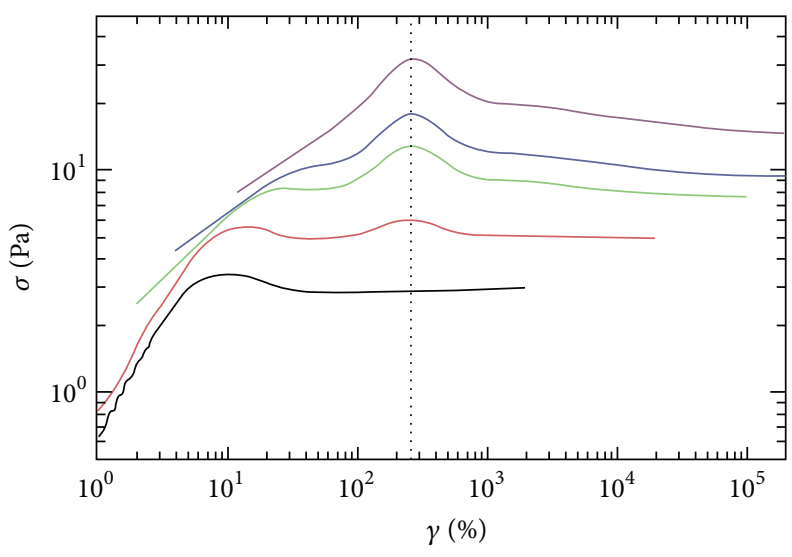

(b)

FIGURE 3: (a) Typical response of shear stress over time for a hypothetical material exhibiting a shear yield stress, at a small, constant shear strain rate. Four different "yield" points are indicated (cf. $[54,73,95])$. The relative magnitudes of the identified stresses, $\tau_{y i}$, may vary. In the dotted profile, two additional yielding events are depicted at much larger values of shear strain. (b) Responses in step rate tests of a depletion flocculated particulate gel (or glass) at $\varphi=0.44$ and $\dot{\gamma}=0.1,1,5,10$, and $30 \mathrm{~s}^{-1}$ (increasing from bottom to top). (Reproduced from Koumakis and Petekidis [60] with permission of The Royal Society of Chemistry.)

also be estimated by analysis of measurements made under oscillatory shear [95].

For some materials, two or even three peaks (or broad humps/shoulders) in the shear stress versus time curve can be observed [59-61] (see also [38]), as in the dotted curve in Figure 3(a) and the experimental results in Figure 3(b). These may be due to transient jamming events that occur even while the structure is breaking up (or densifying) [60, 98]. Alternatively, stick-slip interactions with the wall of the cup may be involved (cf. [99]). The relative height of the individual peaks depends strongly on $\dot{\gamma}$ [59]. Whereas the initial yielding event typically occurs for relatively small strains, the later yielding events can occur at strains on the order of $10^{2}$ to $10^{3} \%$ [59-61].

An alternative configuration of the experimental measurement is to operate in a shear stress-controlled mode [56, 85, 99], effectively a "creep test" [73], rather than strain-controlled as described above. Although claimed to be advantageous for materials sensitive to structural disruption [56] or strain [85], demarcation of the yielding limit is somewhat subjective.

Slip and Associated Considerations. Two of the major reasons for preferring the vane are the minimisation of sample disturbance upon loading and avoidance of depletion effects in which particles or drops migrate away or are excluded from high shear regions and walls, colloquially called "slip" $[46,73,99,100]$. Slip is especially important in yield stress fluids [57].

Slip effects can dominate the results from other techniques unless due care is taken; namely, ensuring tool surfaces are roughened commensurates with at least the particle sizes [101], up to the cluster sizes or even larger [102] (in a particulate gel the clusters are interconnected, and the length scale of surface roughness required on the tool again suggests use of the vane geometry). The phenomenon of slip has still not been fully resolved (see [103]) and is variously reported to occur through a dispersed layer of the colloids or in the suspending fluid alone, at low shear rates or at high shear rates (see also [45]).

In particulate networks, for low shear strain rates, the structure typically fails in a localised region beside one or more of the bounding surfaces, while the bulk undergoes plug flow, resulting in a dominant slip effect $[101,102]-\mathrm{a}$ large change in velocity across the lubrication layer. As $\dot{\gamma}$ is increased, the structure breaks down. While the thickness of the lubrication layer may decrease as the dispersed phase changes from a network to clusters to primary particles, the dominant effect is for a substantial velocity gradient (close to the apparent velocity gradient) to develop in the bulk, so that slip effects eventually become so small as to be neglected.

Most interestingly, in colloidal suspensions, Ballesta et al. [101] reported that the occurrence of slip depended upon normal stresses, with a lower threshold shear stress for slip associated with reduced normal stresses-or, more precisely, greater relaxation of shear-induced normal stress differences.

Recently it has been dramatically reported that shearinduced localised particle depletion is a problem even with vane geometries, in experiments conducted with a concentrated emulsion [46]. The phenomenon reported by Ovarlez et al. [46] is really only of concern in dynamic vane measurements-especially at high shear strains or strain rates-and is expected not to affect static yield stress measurements.

Ovarlez et al. [46] also added to the debate [73] as to whether the boundary of yielding material around the vane can be described by a cylinder or not. Most notably they concluded that, besides the presumed circular Couette flow, extensional flow also occurs, "with [azimuthally]-dependent normal stress differences which have to be taken into account 
in the yield criterion, which thus impacts the [shape of the boundary between yielding and unyielding material]" [46].

At the other interface, between the sample and the wall of the container (e.g., "cup"), slip might also occur, under two circumstances: (i) the local shear stress imparted surpasses the shear yield stress at the wall, although it does not exceed the shear yield stress in the bulk, so that failure occurs only at the wall [99]; (ii) the local shear stress at the wall exceeds the yield value there and also at the periphery of the vane, so that two failure surfaces could occur simultaneously. To date there is no simple remedy for this. The two main options are to roughen the wall or to increase the gap width [99]. Such potential for slip at the outer wall will affect the sample's behaviour upon yielding; it remains to be seen whether a confinement effect could also affect the preyielding behaviour [99] (cf. [57]).

Besides the traditional viscometric techniques, microscopic imaging is becoming increasingly important, including recent advances with confocal microscopy as in Figure 4. Such tools provide direct evidence as to the microscopic reality of the flow, or slip.

\subsection{Normal Stress Differences}

2.3.1. Definitions. In certain complex fluids, normal stresses can be generated under the action of shear flow by mechanisms internal to the fluid. These stresses arise as a direct result of the fluid motion and are not isotropic. The two "deviatoric" normal stresses are described in terms of their difference from the normal stress oriented perpendicular to the shear plane, $\sigma_{22}$. The "first normal stress difference," $N_{1}$, is given by

$$
N_{1} \equiv \sigma_{11}-\sigma_{22}
$$

in which $\sigma_{11}$ represents the normal stress acting in the direction of the shearing/flow (which is frequently considered atmospheric (cf. [104])); the "second normal stress difference," $N_{2}$, is given by

$$
N_{2} \equiv \sigma_{22}-\sigma_{33},
$$

in which $\sigma_{33}$ represents the normal stress acting in the neutral, transverse direction (where stresses are positive in tension) [64]. For simple fluids the normal stress differences are zero, but they take nonzero values for complex fluids-in practice these fluids are found to have an elastic component to their response [64] — and are associated with microscopic anisotropy in the conformation or arrangement of flow units [105].

Rarely a "third normal stress difference," $N_{3}$, is defined [41], given by

$$
N_{3} \equiv \sigma_{11}-\sigma_{33} .
$$

This variable is redundant, as it is equal to simply $N_{1}-$ $N_{2}$, although $N_{3}$ may be a convenient parameter for some applications. Each of the foregoing stresses is sketched in Figure 5.
Besides the normal stress differences themselves, socalled normal stress coefficients are defined as follows [41, 64]:

$$
\begin{aligned}
& \Psi_{1} \equiv \frac{N_{1}}{\dot{\gamma}^{2}}, \\
& \Psi_{2} \equiv \frac{N_{2}}{\dot{\gamma}^{2}} .
\end{aligned}
$$

2.3.2. Mechanism. In polymer solutions or melts, the existence of normal stress differences can be attributed essentially to changes in molecular conformation, and perhaps alignment [41, 64]. In moderate shear environments, when chain scission does not occur, the individual molecules are elastically strained, and they recover their equilibrium conformation when the stress field is removed or dissipated.

In contrast, colloidal particles can be considered perfectly rigid and incompressible, to a first approximation. It is not deformation of the individual particles that governs the response, but rather deformation of the "particulate network" that is established under the influence of interparticle attraction. In that sense, attractive colloidal particle systems initially respond more like solid rubber than like a polymeric fluid. This behaviour is also different to that exhibited by cohesionless granular systems [106], which do not form an elastic network structure. ${ }^{7}$ It is important to emphasise again that the liquid phase is not (directly) involved in these mechanisms for colloidal particle systems; it simply provides buoyancy and an ionic environment. Thus the normal stresses that are of interest herein relate to those experienced by the particulate network itself. Note that, in contrast to the action of the hydrostatic stress, the particulate network in general is not expected to be subject to an isotropic compressive stress (cf. [24]), although this would not affect the analysis unless it resulted in compressive strain (dewatering or densification) simultaneous to the shearing deformation.

The different mechanisms applicable to cohesive networks and cohesionless powders are illustrated by the schematic in Figure 6. In Figure 6(a) the granular system dilates in response to shear; that is, a compressive normal force must be applied externally to counteract the dilatational tendency of the material. (In this system the pore space is typically filled with air, which ordinarily is able to enter or exit the chamber as necessary and otherwise may expand or compress.) By contrast, in Figure 6(b), the colloidal particulate network is stretched in the direction approximately normal to the shearing motion in order to maintain a constant volume. In an open system, ${ }^{8}$ a tensile normal force must be applied externally to counteract the tendency of the material to pull the plates together. Note that Figure 6(b) shows that the gel is expected to still exhibit some elastic properties: that is, at least some of the deformation would be recovered if the stress were relieved; if the material were strained further, irreversible rupture of interparticle bonds would eventually occur.

While the structure of the colloidal particulate network in Figure 6(b) may be reminiscent of the "temporary network model" proposed by Green and Tobolsky [107] to describe 

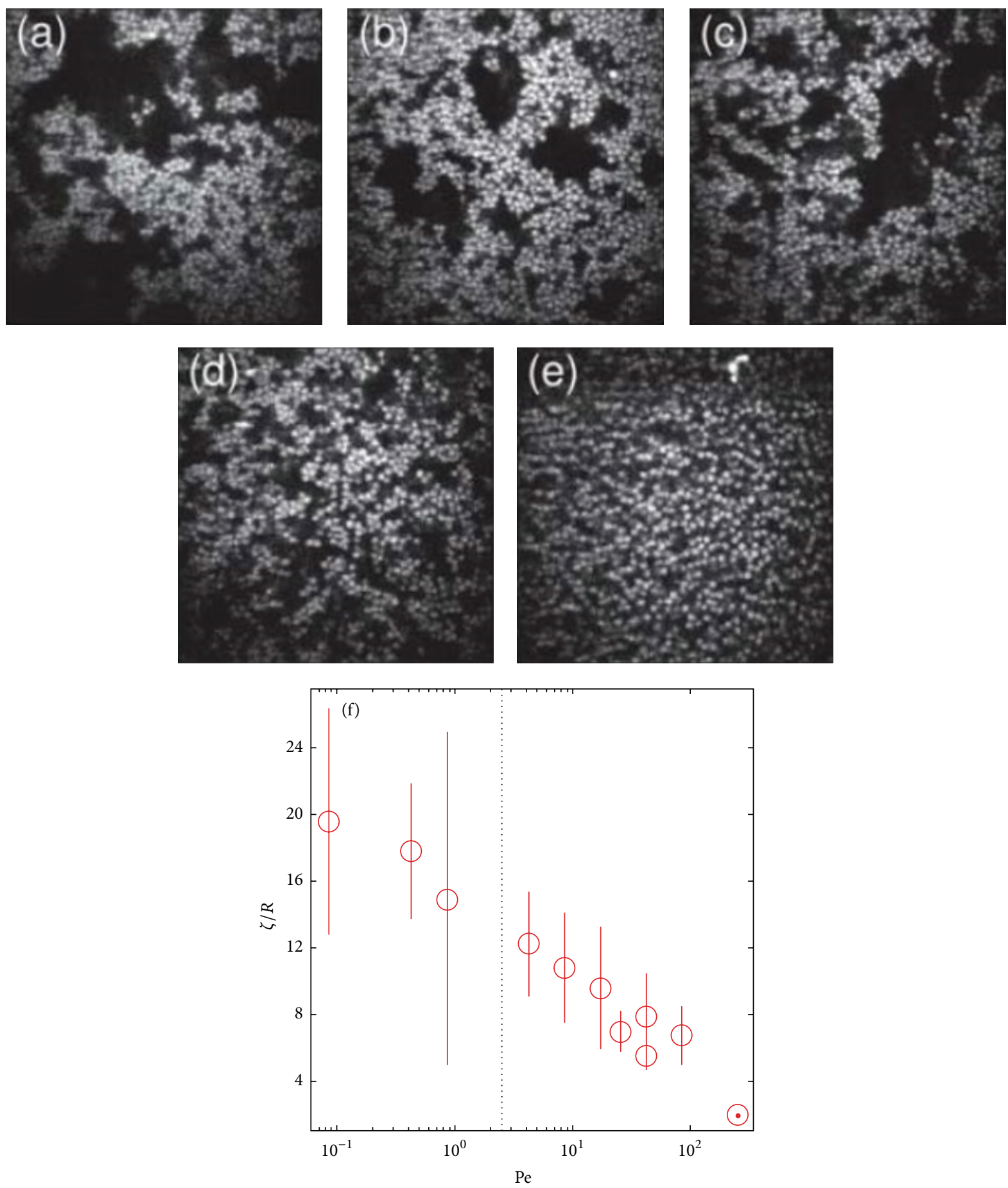

FIGURE 4: (a)-(e) Observations from confocal laser microscopy of a depletion flocculated particle system under shear, with, respectively, $\dot{\gamma}=0.05,0.5,1,5$, and $30 \mathrm{~s}^{-1}$. (f) Nondimensionalised radius of clusters versus applied shear rate (as Péclet number). The dotted line marks the onset of slip. For the system $R=650 \mathrm{~nm}, \varphi=0.4$, and $U_{0}=-20 k_{\mathrm{B}} T$. (Reproduced from Ballesta et al. [102] with permission of The Royal Society of Chemistry.)

polymer melts and the like [41], the key distinction is contained in their description: "temporary." In the polymeric system the nodes of the network represent entanglements that will naturally disengage given sufficient time (other entanglements will then form elsewhere). In contrast, the nodes in the particulate network can be linked by strong interparticle bonds that can only be broken by the imposition of external force.

This explanation might then bring to mind the network structure of, say, a cross-linked solid rubber material. While the junctions between polymer strands for such a material may be enduring [107], indeed the chains themselves are 


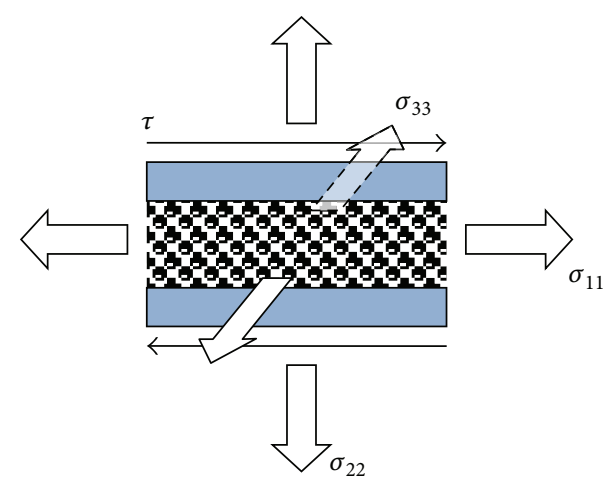

FIgURE 5: Normal stresses, $\sigma_{i i}$, arising during shearing. The sign of the vectors does not correspond to a specific material but is arbitrarily sketched as tension in all directions. The imposed "viscometric" shear stress, $\tau$, is also shown.

expected to endure, and the material is seldom pushed to the point of yielding. Conversely, a shear yield stress of moderate value is characteristic of particulate gels.

Theoretically an inelastic non-Newtonian material could exhibit normal stress effects in steady simple shear flow, but no observations of this are available [64]. Instead the weight of empirical experience has led to the presumption that any normal stress differences observed would be due to elasticity [64] (e.g., [108]).

Influence of Shear Strain and Strain Rate on Normal Stresses. A sample undergoing "pure shear" strain [109-111] must be permitted to contract or dilate in the direction perpendicular to the shear planes (cf. $[93,112,113]$ ), as in Figure 6(a). Conversely, in "simple shear" strain one pair of parallel shearing surfaces is kept at a constant separation $[109,110]$ (cf. [93]), as in Figure 6(b); for a solid-like material this requires an excess normal stress at those surfaces (the Poynting effect) $[113,114]$. For an ideal elastic solid (namely, "neo-Hookean" and incompressible $\Rightarrow$ Poisson's ratio, $v=1 / 2$ ) this would correspond to a compressive contribution to $\sigma_{22}\left(0=N_{2}<\right.$ $N_{1} \propto \gamma^{2}$; that is, $\left.\sigma_{22}=\sigma_{33}<\sigma_{11}\right)[104,113,115]$ (cf. [114]), acting to push the bounding surfaces apart ${ }^{9}[41,105,116]$. This effect is usually negligible for elastic deformations, because of the small strains, $\gamma$, generally involved with colloidal materials $^{10}$ (e.g., $\left.[115,117]\right)$, but may be important in plastic deformation. Given that a shear stress can be expressed in terms of (normal) principal stresses, in turn decomposed into isotropic and deviatoric parts, it is not surprising that Poisson's ratio might play an important role; experimental measurements confirm a very high sensitivity to $v$, including a transition from tensile to compressive $\sigma_{22}$ for very small deviations from $v=1 / 2[104,118]$. In practice the sign of the normal stresses themselves depends on the boundary conditions, or any superimposed isotropic stress [104, 118].

(The cited analyses typically rely on stresses defined with respect to the geometry of the undeformed sample. When large shear strains are contemplated, analysis using contravariant components of the true stress tensor defined on convected or "embedded" coordinates $[113,119]$ may be more appropriate.)
In the "hyperelastic" behaviour just described, the presence of normal stresses at the confining surfaces does not set up any shear or normal strains additional to the simple shear deformation that was imposed. There is no special reason why the normal stress differences could not significantly exceed the magnitude of $\tau$-or indeed $\tau_{\mathrm{y}}$ (cf. [120]).

In contrast to the shear strain, a high shear strain rate is not expected to be required to induce normal stress differences in yield stress materials - unlike polymeric fluids, for which the high shear rates are required to induce alignment, with the stress anisotropy following the structural anisotropy.

\subsubsection{Measurement}

Rheometry. The most intuitive way of measuring normal stresses (and hence their difference) is by means of force transducers mounted on the shaft of a rotational rheometer. This permits direct measurement of $\sigma_{22}$, but by some clever analysis additionally $\sigma_{11}$ can be inferred in a cone-and-plate geometry (see [64]). In torsional flow, using a parallel-plate geometry, Barnes et al. [64] state that the normal stress differences are related to the measured normal force, $F$, according to

$$
\left.\frac{N_{2}-N_{1}}{2}\right|_{\dot{\gamma}_{R}}=-\frac{F}{\text { area }}\left(1+\frac{1}{2} \frac{\mathrm{d}(\ln F)}{\mathrm{d}\left(\ln \dot{\gamma}_{R}\right)}\right),
$$

in which $r=R$ represents the rim of the plate. The lefthand side is just an average of $\left(\sigma_{22}-\sigma_{11}\right)$ and $\left(\sigma_{22}-\sigma_{33}\right)$, evaluated at the strain rate prevailing at the rim, $\dot{\gamma}_{R}$; the first term on the right-hand side is simply $\sigma_{22}$, and the last term is a "correction."

The radial stress in a "cup-and-bob" (or vane) geometry, corresponding to $\sigma_{22}$, could be estimated directly from tappings [121], pressure transducers mounted flush to the surfaces $[64,65]$, or indirectly from calibrated strain gauges (cf. [122]). Commonly $\sigma_{33}$ would be assumed to be zero (i.e., atmospheric pressure acting only), although strictly this should be checked. The assumption is more reasonable for laterally "open" geometries such as parallel-plate but less justifiable for more confined geometries such as capillary flow (cf. [65]).

While direct measurement of normal forces would be most desirable, several potential sources of error exist [57, 64]. Hence, estimation from dynamic oscillatory shear data is often preferred $[64,72]$ (cf. [123]). In the context of yield stress materials, use of steady measurements would surely result in destruction of the particle network (due to the small critical shear strains, $\left.\gamma_{\mathrm{e} 1}\right)$, which can be avoided in small-amplitude oscillatory shear (SAOS).

"Rules" for Viscoelastic Fluids. The conventional analysis relies on an analogy between the steady and oscillatory shear responses in the limit of slow deformation, which is appropriate for the viscoelastic fluids that are usually the subject of investigation. The analysis rests on the assumption that the behaviour of $N_{1}(\dot{\gamma})$ can be inferred from the behaviour of $G^{\prime}(\omega)$, especially in the limit of $\dot{\gamma} \rightarrow 0$ and $\omega \rightarrow 0[41,64]$. This is elaborated in Section 5.1. 

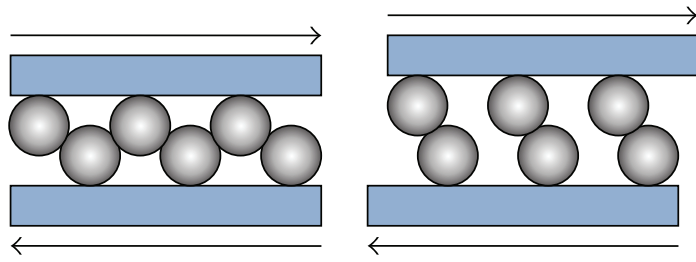

(a)
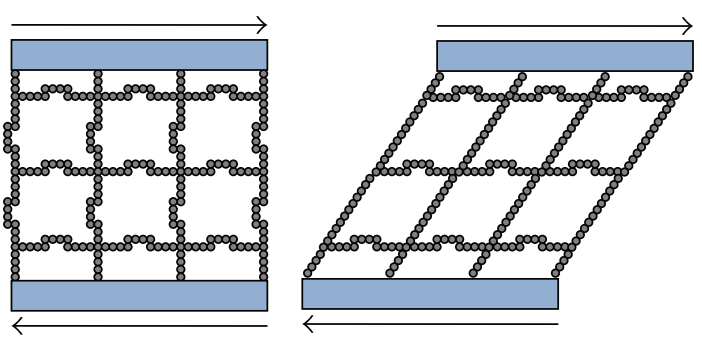

(b)

Figure 6: Schematic of the response of two different types of particle system to shear. (a) Cohesionless granular array (cf. [41]). (b) Colloidal particulate network formed by aggregation (cf. [157]). Normal forces are not shown but are described in the text. It is assumed that wall slip is absent.

Analysis Using Constitutive Models. Generalised analytical results or empirical rules of thumb for viscoelastic fluids may not be applicable to materials that exhibit a yield stress. An alternative approach is to fit the measured experimental shear data to a suitable constitutive model that includes prediction of normal stress differences. Three models that are applicable to yield stress materials are described in Section 5.2.

\section{Potential for Influence of Normal Stress Differences on the Yield Stress}

3.1. Experimental Estimation. The principles by which normal stress differences could affect the experimental estimation of yield stresses are as follows:

(1) The motion of the material as the sample deforms first elastically and then plastically in the apparatus sets up normal stresses differences.

(2) The existence of the normal stress changes the level of shear stress that may be endured before yielding.

(3) Neglecting normal stress differences may also mean that the maximum active shear stress is underestimated.

Whether or not this is significant rests on a number of factors:

(i) Amount of shear strain seen.

(ii) Rapidity with which normal stress differences are established by the shearing.

(iii) Magnitude of the normal stress differences.

(iv) Sensitivity of the yield stress to normal stresses.

In any yielding experiment performed on a real material, some shear strain will occur. It might initially be anticipated that the amount of shear strain will be small, but this is only the case if the measurement technique is intended to operate by gradually bringing an intact specimen to the point of yielding. However, as briefly mentioned above, other measurement techniques exist in which, roughly speaking, the yield stress is determined by analysing the proportions of flowing and nonflowing material as a (not necessarily uniform) stress field acts. The influence of normal stress differences on the yield stress is thus expected to depend on the procedure chosen for determining the yield stress.
Some further comments on the "competing" dynamics of normal stress development and yielding onset are made in Sections 5.2 and 6.1.

It is clear that normal stresses can be influenced by shearing and vice versa. Experimental evidence is consistent with shear yield stresses being influenced by normal stresses, although the sensitivity may not always be large (e.g., [124]) (cf. [125]).

If each of the above conditions is fulfilled, then ignoring the influence would mean that the measured yield stress is misinterpreted as relating to a zero-normal stress condition, when in fact it should be plotted on another part of the "yield surface" - the "envelope," or locus of points in (principal) stress space at which yielding occurs (see, e.g., $[111,113]$ ).

If nothing else, the presence of significant normal stress differences will affect the maximal shear stress experienced in the material, which can be expected (in the first instance) to control the yielding, rather than the shear stress that happens to be oriented (say) perpendicular to the flow direction. Suppose the stress tensor in coordinates defined by the viscometer is given by

$$
\boldsymbol{\sigma}=\left[\begin{array}{ccc}
m & \tau & 0 \\
\tau & n & 0 \\
0 & 0 & 0
\end{array}\right],
$$

where we work in two dimensions for simplicity. Replacing $m-n$ with $N_{1}$, the tensor of principal stresses would then be $[113,126]$

$$
\boldsymbol{\sigma}=\left[\begin{array}{ccc}
\frac{m+n}{2}+\sqrt{\left(\frac{N_{1}}{2}\right)^{2}+\tau^{2}} & 0 & 0 \\
0 & \frac{m+n}{2}-\sqrt{\left(\frac{N_{1}}{2}\right)^{2}+\tau^{2}} & 0 \\
0 & 0 & 0
\end{array}\right] .
$$

The maximal shear stress is thus

$$
\tau_{\max }=\sqrt{\tau^{2}+\left(\frac{N_{1}}{2}\right)^{2}},
$$

in which here $\tau$ is the "viscometric" shear stress imposed perpendicular to the material flow (cf. [92]). 
Of course, whether the maximal shear stress can be interpreted as the threshold for yielding depends upon whether the Mises yield criterion holds or not. This has been tacitly assumed in some theoretical developments [92], perhaps as much for simplicity as anything else. Experimental support for the Mises yield criterion was found by Ovarlez et al. [127] for two different emulsions and a physical gel (Carbopol); however, it should be recognised that these "jammed systems" have entirely different mechanisms of "unjamming" (i.e., yielding), namely, deformation of the dispersed phase elements, than do colloidal gels, in which bond breakage (or at least sliding) is needed. It is typically very difficult to conclusively distinguish the form of the yield stress surface from only a small number of data points (e.g., [128]). The yield envelope of real materials is liable to take on a more complicated form than posited by the classical theories (Mohr-Coulomb, Tresca, and Mises) [129, 130], and it is simplistic to assume that any one of these yield criteria is "normal." Finally, the yield stress manifold is in principle different for the distinct cases of static yield and dynamic yield [131]. For these reasons, support for a Mises yield criterion in "soft glassy materials" in general, and especially in attractive colloidal particle networks, should be treated as preliminary or approximate until more comprehensive data is at hand (cf. [132]). Of special interest in the present context is that a Mises yield criterion is deduced only to apply exactly in the absence of normal stress differences [127, 131].

A separate problem, already alluded to, is the possibility that the dimensions of the flowing portion of a sample may differ substantially from those otherwise assumed [46]. If not accounted for, this would result in misestimation of the shear yield stress. Unfortunately, it is not easy to account for.

3.2. Application. The larger problem is that the purpose of measuring, or otherwise estimating, shear yield stresses is ultimately for their use in some application. That application may involve equipment design, or process optimisation, for example. In some disciplines (e.g., soil mechanics) the expected and desired state of the material may be static, and that might correspond neatly to the experimental measurement regime. On the other hand, in chemical engineering applications, it may be intended that the material flows. These sorts of conditions may produce more significant nonlinear effects, including normal stress differences, than are encountered in simpler rheometric flows [133].

Especially in complex industrial flows, the stress state of the material in the application is probably different to the state prevailing during the measurement. In other words, the locus of the stress state with the yield envelope would be different in the two cases. Furthermore, this would suggest the yield envelope itself may evolve differently as the yielding progresses. A better knowledge of the normal stress differences is therefore needed [134].

The situations encountered in industry include any of those shown schematically in Figure 7. Of most interest to the present discussion are those in which the elastic network response dominates, at least in a significant part of the volume as in Figure 7 (f) (cf. $[135,136])$, if not throughout the entire volume as in Figure 7(g).
3.3. Inference. The preceding discussions have dealt with complications in the analysis of rheometric or arbitrary flows. A further motivation for measuring normal stress differences is that by monitoring the normal stresses during, say, a vane test experiment, it may be possible to get greater insight into structure development (or breakdown) than by monitoring the shear stress alone (cf. [137]).

\section{Observations of Normal Stress Differences in Aggregated Colloidal Systems}

Most of the study of normal stress differences has been directed toward polymer systems, with few data available in the literature for colloidal and particulate materials [72, 105, $138,139]$. The extant data mostly indicate $0 \lesssim-N_{2} \ll N_{1}$ (i.e., $\left.\sigma_{22} \lesssim \sigma_{33} \ll \sigma_{11}\right)[41,64]$, but this generalisation may not be applicable to particulate systems, for which $N_{2} \lesssim N_{1} \ll 0$ has also been reported (i.e., $\sigma_{11} \ll \sigma_{22} \ll \sigma_{33}$ ) [105, 139] (cf. $[131,138])$. The limited data on suspensions refers mostly to materials in which there is little or no aggregation of the particles, as depicted in Figures 7(b), 7(c), and 7(e). (This is distinct from ephemeral clusters arising due to hydrodynamic forces, or "hydroclusters" [98, 134].) In these dispersed colloidal systems undergoing steady simple shear, the magnitude of $N_{1}$ becomes significant at higher solid fractions in the concentrated regime, approaching random close packing, being comparable to the prevailing shear stress with $\mathcal{O}\left(N_{1} / \tau\right) \sim$ $10^{-1}$ to $10^{+1}[105,138,139]$. The few available publications that report normal stress differences for coagulated or flocculated systems relate mostly to weak, reversible flocculation, with similar magnitudes of $N_{1} / \tau[116,140,141]$, whereas strongly aggregated colloids may have quite different properties [140].

Key results from Otsubo [140] are presented in Figure 8. For each of the two solids concentrations the normal stress differences only become significant at higher shear strain rates. This already suggests that the normal stress differences were generated by shear-induced alignment of individual flocs, rather than by an intact particle network. That diagnosis is supported by the fact that the measurements were carried out in steady shear (in a cone-and-plate viscometer), so that the accumulated shear strains can be expected to be beyond the applicable yield strain.

A few research groups have recently reported normal stress differences for aggregated suspensions [120, 142, 143]. However, the shear strains and strain rates imposed during the measurement tended to be large, so that the material response would not be that of a persistent particle network, but rather of dispersed flocs in brief and intermittent contact, of the sort sketched in Figure 7(d) or, possibly, Figure 7(f). In such configurations, the solid phase does not resist much of the imposed shear stress, in comparison to an intact particle gel as sketched in Figure 7(g), in which the solid phase alone can resist the whole shear load. Indeed the flocs described by those researchers are often weak, sometimes even being formed purely by hydrodynamic forces. Besides the large strains imposed during measurement, often the experimental protocol involved subjecting the samples to high shear prior 


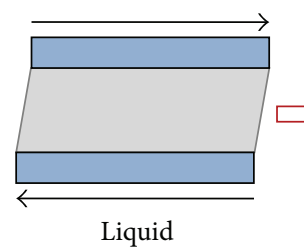

(a)

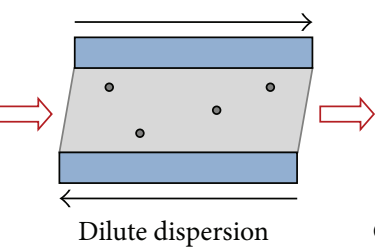

(b)

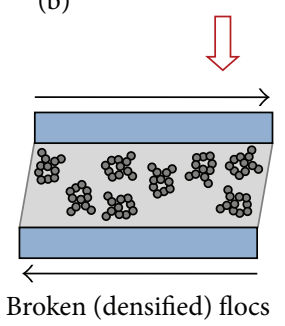

(d)

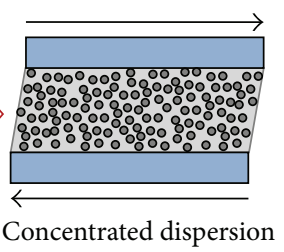

(c)

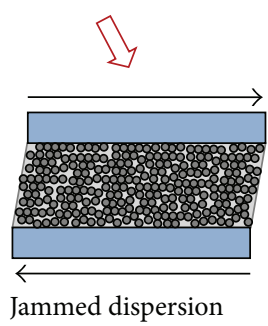

(e)

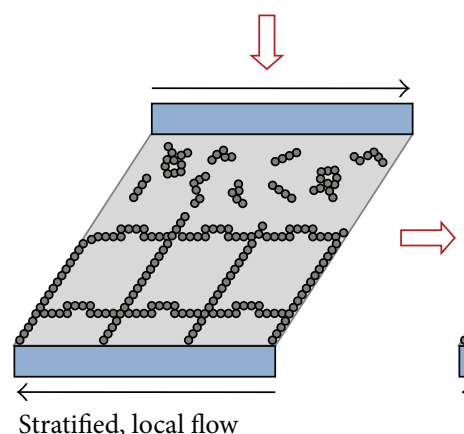

(f)

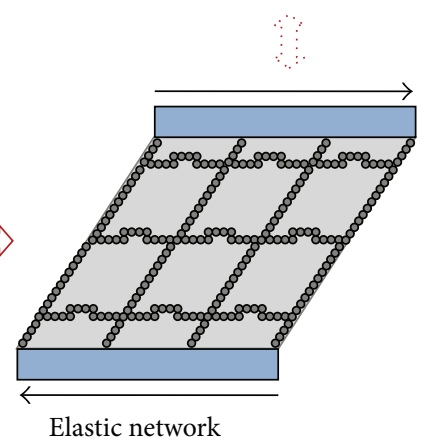

(g)

FIGURE 7: Schematic of different physical flow behaviours of repulsive or granular particles (dispersed) and attractive particles (aggregated) of various concentrations, in response to imposed shear. Arrows indicate increased ordering of particles.

to measurement, without mention of any pause for relaxation and possible network reformation [120], greatly affecting the material properties (see also [15, 144, 145]); the steady shearing also led to macroscopic structuring, promoting lubrication stresses, different to the original, macroscopically homogeneous gel. A potential complication with the work of Lin-Gibson et al. [142] is their use of polyisobutylene as the carrier fluid; these polymers are generally viscoelastic [146].

For completeness it should be recognised that an isotropic component to the flow-induced normal stresses acting on the solid phase can also arise in dispersed particle systems $[105,139]$. Considering the physical mechanisms proposed to contribute to this effect, and following from an absence of experimental data to the contrary, we consider that this isotropic parameter will be less relevant to aggregated systems than the normal stress differences.

\section{Models for Normal Stress Differences}

To date there are no models that have been specifically developed to predict normal stress differences in aggregated colloidal systems, and so recourse must be made to generic models.

5.1. Relations for Viscoelastic Fluids. The most well-characterised rheological fluids are polymer melts [61], and a starting point is to consider the applicability of relations originally derived for polymer melts to aggregated colloidal systems.

A commonly cited (e.g., [41, 64]) relation was derived exactly by Coleman and Markovitz [147] for a "second-order fluid," as an approximation to the deformation of a general simple fluid with fading memory (see [65]), in the zero-shearrate limit:

$$
\lim _{\dot{\gamma} \rightarrow 0} \Psi_{1}(\dot{\gamma}) \equiv \lim _{\dot{\gamma} \rightarrow 0} \frac{N_{1}(\dot{\gamma})}{\dot{\gamma}^{2}}=\lim _{\omega \rightarrow 0} 2 \frac{G^{\prime}(\omega)}{\omega^{2}} .
$$

Further discussion of (9) and other related formulae is presented in the Supplementary Material.

It would be of interest to compare the normal stress differences with the prevailing shear stress that might apply in the limit of small $\dot{\gamma}$ for nonoscillatory flow. The shear stress is found from [64]

$$
\tau=\eta \dot{\gamma}
$$

Hence

$$
\lim _{\dot{\gamma} \rightarrow 0} \frac{N_{1}(\dot{\gamma})}{\tau}=\lim _{\omega \rightarrow 0} 2 \frac{G^{\prime}(\omega)}{G^{\prime \prime}(\omega)} .
$$

Although approximate, there is at least a semblance of logic in (11), with the ratio of normal stresses to shear stress being 


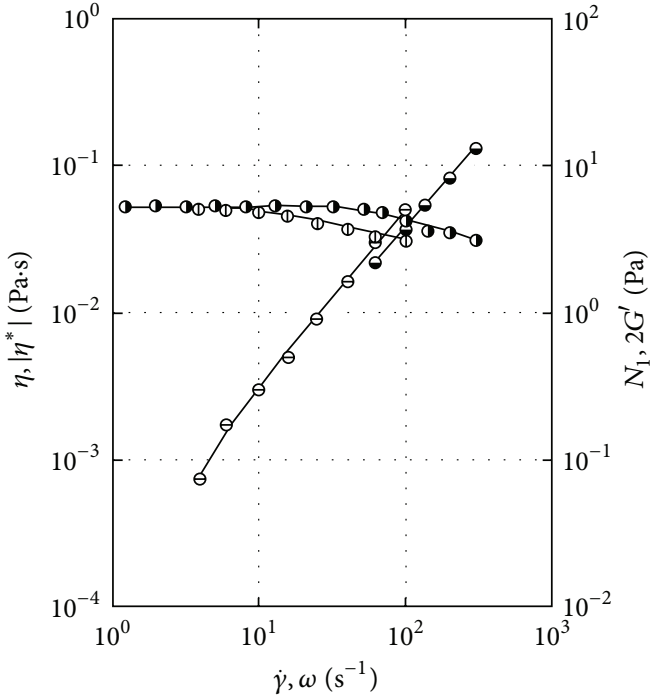

(a)

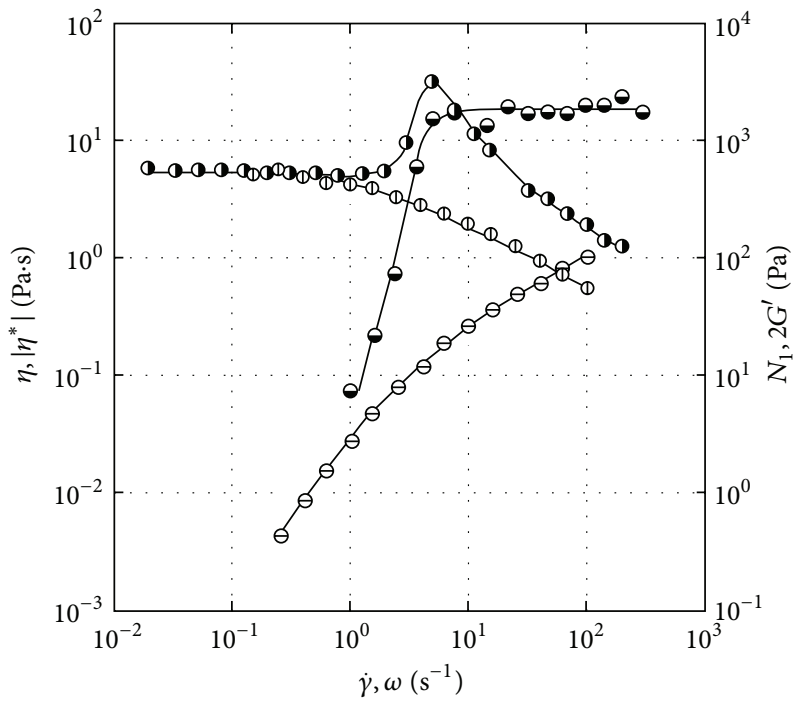

(b)

FIGURE 8: Response of polymer-flocculated $80 \mathrm{~nm}$ copolymer particles for (a) $\varphi=0.05$ and (b) $\varphi=0.20$. Viscosity ( $\mathbf{(})$ and first normal stress differences $(\ominus)$ in steady shear and magnitude of complex viscosity $(\Phi)$ and storage modulus $(\ominus)$ in oscillatory shear. (Reprinted from Otsubo [140], with permission from Elsevier.)

proportional to the ratio of elastic and viscous response components.

A more general version of (11), applicable at any shear rate, has been separately suggested on theoretical and experimental grounds, for polymeric fluids, being known as Lodge's rubberlike liquid theory [148]. This yields [148]

$$
N_{1}=2 \tau \gamma_{\mathrm{r}, \mathrm{s}}
$$

in which $\gamma_{\mathrm{r}, \mathrm{s}}$ is the steady-state recoverable (i.e., elastic) shear strain.

Alternative arguments and experimental data for polymer solutions and melts yield a formula that differs by a factor of two; namely [148],

$$
N_{1}=\tau \gamma_{\mathrm{r}, \mathrm{s}}
$$

A variety of other superposition techniques have been proposed for other applications (e.g., [66, 67, 149]). More research is required to determine which of these superposition techniques-if any-can be applied to attractive particulate systems.

5.2. Constitutive Models for Yield Stress Materials. As viscoelastic fluids do not exhibit a yield stress, we next present details of three models that provide for a yield stress alongside shear-induced normal stress differences both above and below the nominal yield stress. While each model contains an array of adjustable material parameters, the tendencies described below are inherent to the models irrespective of material parameter values.

5.2.1. Saramito's First Model. Saramito [47] introduced a true elastoviscoplastic macrorheological model, constructed from a combination of four basic elements (cf. [150]). Before yielding the model material behaves as a viscoelastic KelvinVoigt object, while above the yield stress the material behaves as a power-law viscoelastic fluid following Oldroyd's theory. Further features of the model are presented in the Supplementary Material.

For relatively large values of the Bingham number, that is, for large values of the threshold stress and/or at small values of the viscous resistance (slow oscillation, small amplitudes, or low viscosity), the ratio of first normal stress difference to shear stress can be estimated. Under oscillatory shear below the critical stress we obtain

$$
\begin{aligned}
& \frac{\operatorname{mean}\left\{\left|N_{1}(t)\right|\right\}}{\operatorname{mean}\{|\tau(t)|\}} \approx \begin{cases}\frac{1}{2} & \text { for small }|c| \\
\frac{\pi}{4}|c| & \text { for large }|c|,\end{cases} \\
& \frac{\max \left\{\left|N_{1}(t)\right|\right\}}{\max \{|\tau(t)|\}}=\frac{|c|}{2}+\frac{1}{2}, \quad \forall c,
\end{aligned}
$$

in which $t$ is time and $c$ is an "arbitrary constant" dependent upon the initial stress state (cf. [92]).

These ratios show that the first normal stress difference is predicted to be at least comparable to the shear stress, and may in principle be many times greater. Note also that the rate or magnitude of the shear strain does not enter into these equations.

Saramito's model does not expressly indicate the magnitude of $N_{2}$.

For moderate values of the Bingham number, solution is only feasible by numerical methods. To provide a sense of the transition, an analysis of the predictions for a Bingham number of zero is provided here. This case corresponds to the opposite limit from that taken above: it is reached for 


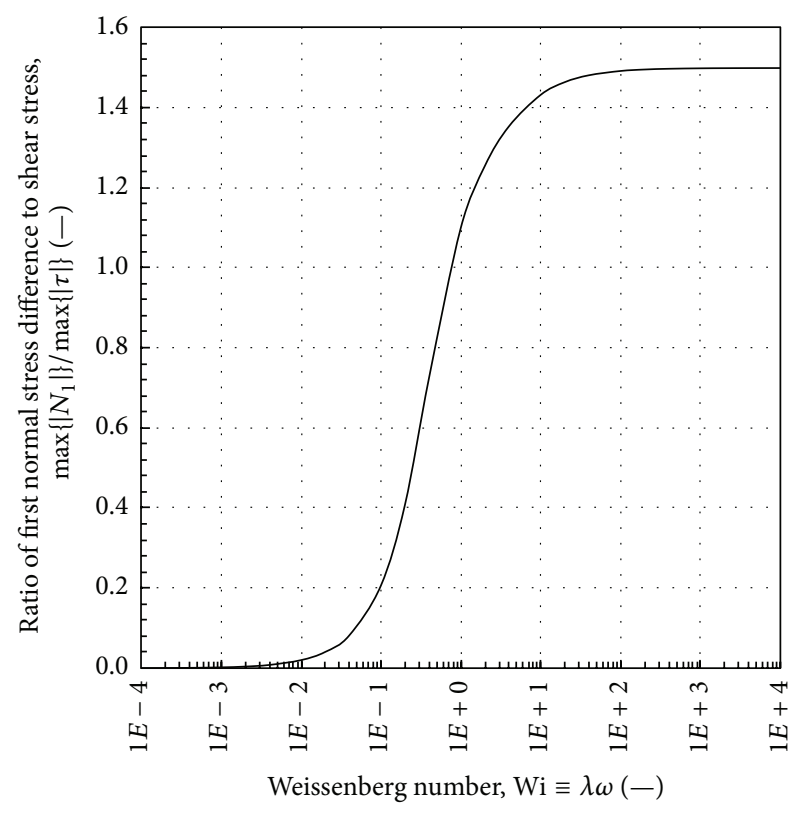

FIGURE 9: Ratio of maximum first normal stress difference to maximum (nominal) shear stress magnitudes experienced in oscillatory simple shear flow, as a function of the Weissenberg number, in the limit of zero (or very small) Bingham number, according to Saramito's first model.

very large amplitude oscillation, although it is also attained for negligible threshold shear stress, for very high frequency oscillation, or when the viscosity is very high. (In all of these conditions the viscous resistance controls the response.) By taking a ratio of the maxima (as in (15)), it is possible to obtain an indicative ratio of the first normal stress difference to the nominal shear stress as a function of the Weissenberg number, as presented in Figure 9. The Weissenberg number may here be defined as $\mathrm{Wi} \equiv \lambda \omega$, in which $\lambda$ is the material's relaxation time, and $\omega$ is the frequency of the imposed oscillation [47]. Recalling that, in the limit of large Bingham number, (15) predicted a ratio of maximum stresses of order 0.5, the data of Figure 9 suggest that as the Bingham number is reduced, the stress ratio may increase somewhat at moderate to high values of $\mathrm{Wi}$, whereas it would be expected to decrease at low values of Wi.

Precisely which ratio applies for a given experiment will depend upon both the material properties and also the shear regime to which it is exposed (not to mention the initial stress condition).

Saramito [47] also simulated the transient stress development under simple shear at a constant rate. Although the shear stress commenced at a magnitude greater than the first normal stress difference, the growth in $N_{1}$ was more rapid, and ultimately the magnitude of $N_{1}$ exceeded $\tau$ for the given conditions.

These results reinforce the importance of considering the influence of normal stresses not only upon the material response prior to yielding, but also on the "steady-state" behaviour.
5.2.2. Model of Doraiswamy et al. ${ }^{11}$. Doraiswamy et al. [91] presented a model for a material that would deform elastically below its yield stress and flow viscoplastically above $\tau_{\mathrm{y}}$. The model is composed of Hooke's equation below a critical shear strain (corresponding to $\tau_{\mathrm{y}}$ ), and the Herschel-Bulkley equation above the critical shear strain. Further features of the model are elaborated in the Supplementary Material.

It can be shown that, below the yield stress, the ratio of the first normal stress difference to the shear stress under oscillatory shear is approximately

$$
\frac{\max \left\{\left|N_{1}(t)\right|\right\}}{\max \{|\tau(t)|\}}=\frac{\operatorname{mean}\left\{\left|N_{1}(t)\right|\right\}}{\operatorname{mean}\{|\tau(t)|\}} \sim \gamma_{0} .
$$

This is also predicted by the Lodge-Meissner "rule" [133] (cf. (12) and (13)). Given the small value of the shear strain amplitude, $\gamma_{0}$, expected prior to yielding (it must be no more than $\gamma_{\mathrm{c}}$ ), the first normal stress difference is predicted to be small in comparison to the shear stress, in contrast to the predictions obtained by examining Saramito's first model.

The model of Doraiswamy et al. predicts $N_{2}=0$ for the above conditions.

5.2.3. Saramito's Second Model. In later work, Saramito [63] explored the combination of a Kelvin-Voigt response below the critical shear stress with a viscoelastic response at higher stresses based on the Herschel-Bulkley equation. That is, he effectively replaced the Oldroyd equation with a power-law equation. The resulting system is similar to the model of Doraiswamy et al. [91], except that viscous deformation is permitted below the threshold stress. Further features of the model are discussed in the Supplementary Material.

Just as in Saramito's first model, his second model predicts that when exposed to simple shear at a constant rate the magnitude of $\tau$ is initially greater than $N_{1}$, but the transient growth in $N_{1}$ is more rapid, and ultimately the magnitude of $N_{1}$ exceeds $\tau$ for the given conditions [63]. Notably, in the initial period where the shear stress is less than the threshold value, $\tau$ increases linearly with time, while $N_{1}$ increases quadratically - that is, the material behaves as an elastic solid, obeying the Poynting law $[63,92]$. This is consistent with the first-order and second-order dependence of $\tau$ and $N_{1}$, respectively, upon shear strain for application of a constant shear rate with the model of Doraiswamy et al. (see Supplementary Material, equations 23 and 26). An analogous result has recently been reported for circular Couette flow [92].

The newer analyses also propose some surprising phenomena, whose practical effect is to cast further doubt over results obtained in the presence of normal stress differences. By applying Saramito's second model to circular Couette flow, it was discovered that the radial normal stress that existed upon loading would persist perpetually in the unyielded material around the outer wall of the cell; hence even steadystate flows are nonunique [92]. Meanwhile, experimental and numerical modelling results showed that $N_{1}$ could change sign across the annular gap due to evolution of the azimuthal normal stress [92]. The strong dependence of the measured response upon the initial stress condition of the loaded sample was proposed to explain much of the experimental 
nonreproducibility reported in the literature for materials such as foams, emulsions, and colloidal gels [92].

Cheddadi et al. [92] went so far as to ask whether Couette flow was at all desirable for the purpose of characterising elastoviscoplastic materials. They concluded that the flow regimes that occur would make interpretation of results difficult, especially due to the dependence upon the initial stress condition prior to shearing, as per Figure 10. It was suggested that a "high velocity preshear in the reverse direction" could serve to "neutralise" normal stresses in the original sample.

It should be recognised that in a "vane" measurement of the shear yield stress, most of the material is static. According to the above analysis, this would mean that the poorly reproducible stress history of the sample would have a strong effect on the resulting material response. As alluded to in Section 2.1.2, for aggregated colloidal systems, it is not appropriate to conduct vigorous preshearing without time allowed for reequilibration, because the material properties are likely to change. Once the particulate network is broken, even after resting, it may not be able to reform the original structure, for example, due to "ageing" effects [24, 151].

These results imply a possible influence of normal stresses not only upon the material response prior to yielding, but also on the "steady-state" behaviour. The findings also have implications for "validation" efforts, insofar as a measurement of the radial normal stress is insufficient to draw conclusions regarding $N_{1}$ : it would be necessary to know the azimuthal normal stress too.

\section{Discussion}

6.1. Dynamic Behaviour. Although it is often written that $N_{1}=N_{1}(\dot{\gamma})$ [41, 64] (cf. [139]), little is known about how the normal stresses would develop-or relax [143, 152]—in practice. Again, more data is available for polymer melts (e.g., $[41,148]$ ) and polymer solutions (e.g., $[153,154])$, although even for these materials a diverse range of behaviours has been reported. For strongly aggregated particulate networks we expect that the evolution would depend more on shear strain than on the shear strain rate (cf. [144]), because these materials are not able to relax the elastic strain unless the imposed stress is removed (cf. Figure 6(b)). Estimates of $\tau_{\mathrm{y}}$ made by monitoring the peak shear stress obtained while rotating a vane at constant shear strain rate [90] might also be somewhat affected. Other essentially "static" measurement techniques can also be employed to obtain direct estimates of $\tau_{\mathrm{y}}$ by observing the onset of plastic flow [90] and would be less affected by normal stress differences-aside from those due to sample preparation and loading.

As mentioned earlier, it is not just in measurement scenarios that dynamic behaviours should be considered, but also in the material applications. In practical applications, the reestablishment of a gelled phase in low shear regions may also be important, for which the possible influence of normal stress differences has not been investigated up to now.

6.2. Ramifications. The effect of $N_{1}$ on the shear yield stress has not been previously considered. It is apparent that the stress tensor that includes normal stresses arising due to shear flow will not be "similar" in form to the stress tensor that is obtained by the simple superposition of shear flow in an "undrained" pressurised system. Any pressure imposed in such a system would be borne predominantly by the liquid phase (cf. [139]), due to its much greater bulk modulus compared to that of the particle network (see [24]). As with the particulate network stresses found in dewatering processes, the different normal stresses arising as a consequence of shear flow are associated exclusively with the solid phase, because the liquid phase can accommodate only isotropic stresses [139] (ignoring polymeric liquids). The combination of stresses seems even to be different from that applicable in experiments such as those of Channell [124], in which a shear field is superimposed on a filtration operation (i.e., "drained"), due to differences in the lateral stresses relative to the axial stress. In any case, it does not seem to be correct to identify $N_{1}$ as equal to the compressive yield stress (in the absence of shear)_even if only "numerically" — as implied in the "yield surface" specified by Holmqvist and Dahlkild [155].

It is of interest to consider how normal stress effects can be distinguished. Ideally the models would be developed to an extent that we would know the normal stresses arising in various geometries and experimental configurations, so that yield stresses estimated under the action of diverse normal stress differences could be analysed that way. Currently the need is for more experimental data, in which normal stress differences are directly estimated. In an iterative process these will inform the model development. Meanwhile, yield stress measurements by a range of procedures will sometimes permit experimental assessment of normal stress differences, but in some cases the experimental setups make measurement of normal stress differences impossible. Some experimental investigations have shown comparable estimates of the shear yield stress using different techniques. It can be that in specific cases either (i) the normal stress differences are small, (ii) the effect of the normal stress differences on the yield stress is small, or (iii) the effects of the normal stress differences on the yield stress are similar in two different experimental configurations. In working with weakly aggregated materials, thixotropy may also be an issue. To a first approximation, normal stress effects are proposed to be functions of strain, rather than strain rate. Time-dependent effects could then be explored by observing the yielding transition at different experimental timescales.

6.3. Yield Stress and Yield Strain. One of the recurrent questions in the field of rheology is whether any material exhibits a "true" yield stress. As described earlier, practical definitions of yielding delineate the behaviour of ordinary flow and "creep" or demarcate specific yielding events. Even though the plastic deformation is not identically zero below these yield stresses, and some form of flow can be observed, the particulates remain more-or-less interconnected.

It is expected that, to attain significant normal stress differences, commensurate shear strains would have to be attained. The foregoing analysis reveals that even where a sample is nominally considered "brittle" due to a low yield strain, in practice two features can contribute to increasing the strain actually developed. First is flow by "creeping" below 


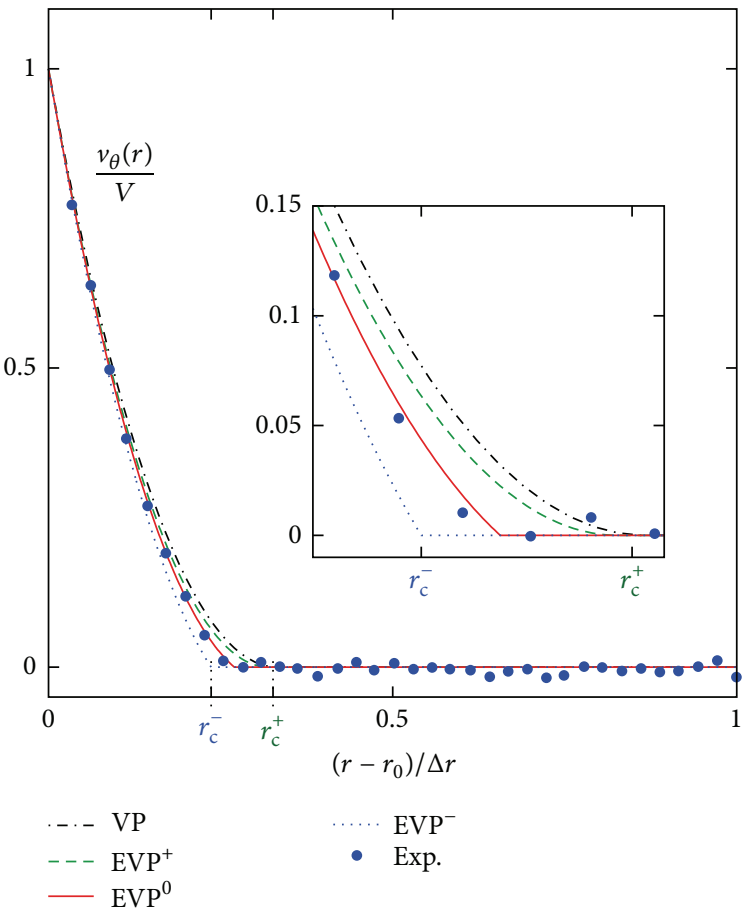

(a)

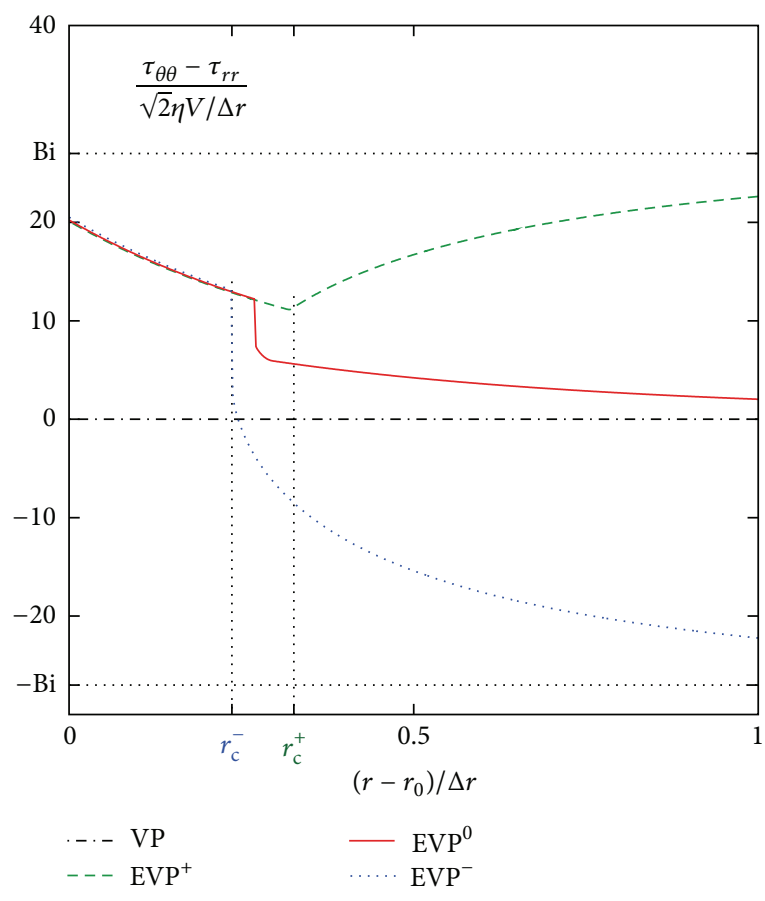

(b)

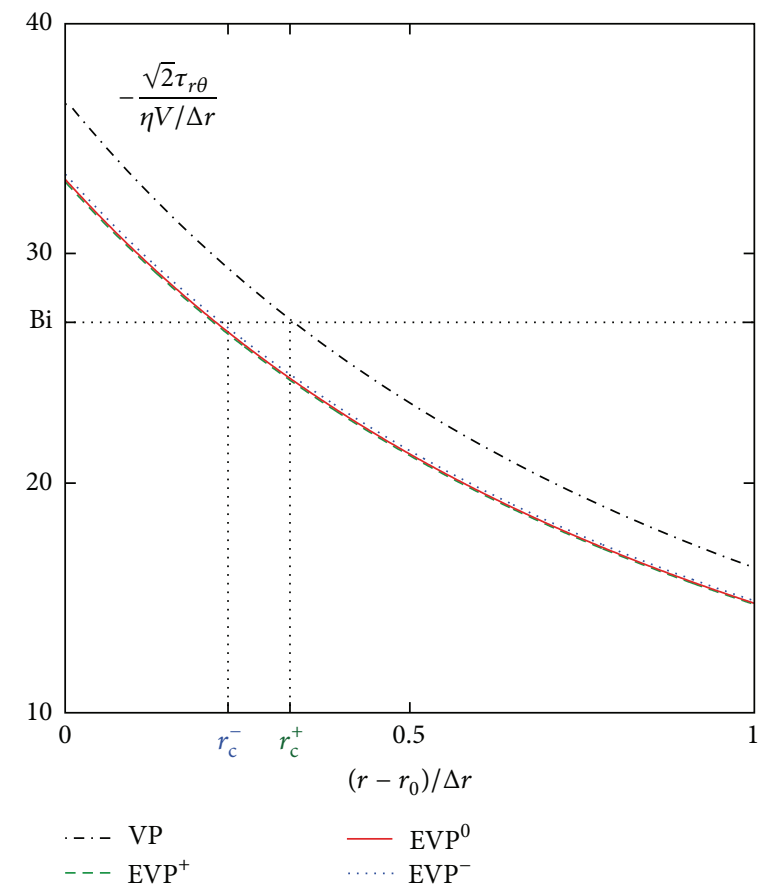

(c)

FIGURE 10: Comparison between the steady-state solutions of the viscoplastic (VP) Herschel-Bulkley model and the elastoviscoplastic (EVP) Saramito model in cylindrical Couette geometry with different residual stresses: (a) velocity, with experimental data (exp.) for a natural bentonite suspension (from [136]); (b) normal stress; (c) shear stress. Lines: solutions of the EVP model with different initial stress conditions. Thick solid line $\left(\mathrm{EVP}^{0}\right): \tau_{\theta \theta}=0$; thin dashed line $\left(\mathrm{EVP}^{+}\right): \tau_{\theta \theta}=+\sqrt{2} \tau_{\mathrm{y}}$; thin dotted line $\left(\mathrm{EVP}^{-}\right): \tau_{\theta \theta}=-\sqrt{2} \tau_{\mathrm{y}}$; thick dash-dotted line $(\mathrm{VP}) . r_{\mathrm{c}}$ represents the critical radius for flow. (Reprinted with permission from Cheddadi et al. [92]. Copyright 2012, The Society of Rheology.) 
the lowest yield stress criterion. Hence even materials with merely "apparent" yield stresses are subject to interdependence of the shear and normal stresses. This was allowed for in the two models of Saramito, which admitted creeplike deformation even below the threshold shear stress. The second feature is the existence of secondary or tertiary yield stress criteria, corresponding to much larger shear strains. Under these conditions it is natural for significant normal stress differences to occur. Large shear strains at incipient yielding have also been reported from experimental measurements on strongly coagulated and strongly flocculated colloidal suspensions, for example, shear yield strains of order 1 [99].

\section{Conclusion}

Attractive particle networks comprise a class of materials that manifest complicated characteristic responses to imposed loads or imposed deformation. Normal stress differences are more familiar in elastic or viscoelastic materials. Yield stress materials, such as particulate gels, are commonly assumed to be rigid prior to yielding; however, they are more accurately modelled as elastic prior to yielding, so that normal stress differences can be generated upon shearing. Normal stress differences of magnitude comparable to the nominal shear stress are predicted in some models and supported by experimental data. Such large normal stress differences may impact the behaviour and characterisation of yield stress materials in two ways. First, the shear yield stress may be a function of the normal stress differences, in which case it is important to know the full tensorial stress state at yielding (cf. [92]), in order to correctly locate the information upon the "yield surface." Second, the maximum shear stress experienced in the material may not align with the geometry of the walls and will be underestimated in the presence of normal stress differences if these are not accounted for. As this topic has received very little attention to date, opportunities remain to gather more experimental data and to further develop the applicable models.

\section{Competing Interests}

The authors declare that they have no competing interests.

\section{Acknowledgments}

The authors thank The University of Melbourne for access to their facilities. The authors express their gratitude to Professor Peter Scales for his support in their separate endeavours while at the University. David Verrelli wishes to acknowledge a travel grant in memory of the late Associate Professor Howard See that was awarded by the Australian Society of Rheology, which aided in the dissemination of an early incarnation of this work at the 2011 Korean-Australian Rheology Conference. Dr. Verrelli also thanks Marcus Zipper and CSIRO Process Science and Engineering for support. Finally, the authors acknowledge helpful correspondence with Andrew Chryss, Ronald Larson, Yasufumi Otsubo, and Woojoo Han.

\section{Endnotes}

1. While the stereotypical colloidal network is often well represented as an equilibrium system (at least while not being deformed), biological systems are more likely to comprise out-of-equilibrium systems-specifically, "active colloids" [32]. Biomimetic synthetic objects use microtechnology and nanoparticles to replicate some of the behaviours of naturally occurring microorganisms [29].

2. The concept of a threshold stress to produce plastic (irreversible) yielding goes back much further, at least to Maxwell circa 1871 [158] (cf. [52]), and arguably to Tresca in 1864 , or perhaps earlier (see [159]).

3. The attraction and network formation may be a result of coagulation, flocculation, magnetic forces (e.g., [160]), or some other means (cf. [161]); those details do not affect the present analysis, provided that any external fields that affect the particle interactions (e.g., $[54,162]$ ) are maintained constant or zero.

4. This demonstrates that the $\tau_{\mathrm{y} 3}$ condition does not require that the "majority of the particle-particle bonds are broken" (cf. [95]), although we can expect that it corresponds to the minimum number of particle-particle bonds occurring in the sequence.

5. The first "rheological" use cited by Barnes and Nguyen [73] was in 1936.

6. It is generally accepted that this phenomenon is not "true" slip of the sample [100], because the velocity varies continuously through the embedding medium, from zero at the wall up to the apparent slip velocity; however, when depletion proceeds far enough that there is a layer of fluid that is entirely free of the dispersed phase, then the dispersed phase could be said to lose contact with the opposing boundary, and this could reasonably be described as true slip with respect to the dispersed phase only.

7. Nevertheless, for very small strains (smaller than the primary particle size), the deformation of a cohesionless particle system, such as sand, would still be reversible; the main difference is that the particles themselves would not "drive" the recovery, but rather the system would rely on external forces, such as the applied shear stress being reversed, or gravitational potential.

8. The system as a whole is practically incompressible when undrained; in the drained condition the network compressibility is isolated, as the (incompressible) liquid is allowed to drain out-corresponding to dewatering [24] —or even to flow into the system; in a closed system the pull on the plates by the network may be resisted by an elevated superimposed (isotropic) pressure throughout the sample.

9. This is opposite to that presumed for the idealised particulate force chains sketched in Figure 6(b).

10. Within elastic solids, the normal stresses set up in some cases of shear deformation could lead to changes in 
volume, if not resisted, and potentially progress to failure $[104,118]$; these underlying stresses could be a useful way to describe the dilatancy upon shearing that is a feature of "critical state" soil mechanics [93] —or general shearenhanced volumetric strain [113] —although this link does not appear to have been investigated previously.

11. This may alternatively be referred to as the "RutgersDelaware model" (cf. [163]).

\section{References}

[1] J. Mewis and N. J. Wagner, Colloidal Suspension Rheology, Cambridge University Press, Cambridge, UK, 2012.

[2] G. Lois, J. Blawzdziewicz, and C. S. O’Hern, "Jamming transition and new percolation universality classes in particulate systems with attraction," Physical Review Letters, vol. 100, no. 2, Article ID 028001, 2008.

[3] A. A. Potanin, "On the computer simulation of the deformation and breakup of colloidal aggregates in shear flow," Journal of Colloid and Interface Science, vol. 157, no. 2, pp. 399-410, 1993.

[4] G. V. Franks and F. F. Lange, "Plastic-to-brittle transition of saturated, alumina powder compacts," Journal of the American Ceramic Society, vol. 79, no. 12, pp. 3161-3168, 1996.

[5] B. V. Velamakanni, J. C. Chang, F. F. Lange, and D. S. Pearson, "New method for efficient colloidal particle packing via modulation of repulsive lubricating hydration forces," Langmuir, vol. 6, no. 7, pp. 1323-1325, 1990.

[6] D. J. Shaw, Introduction to Colloid and Surface Chemistry, Butterworth-Heinemann, Elsevier Science, Oxford, UK, 4th edition, 1992.

[7] R. J. Hunter, Foundations of Colloid Science, Oxford University Press, Oxford, UK, 2nd edition, 2001.

[8] J. N. Israelachvili, Intermolecular and Surface Forces, Academic Press, Elsevier, Amsterdam, The Netherlands, 3rd edition, 2011.

[9] W. B. Russel, D. A. Saville, and W. R. Schowalter, Colloidal Dispersions, Cambridge University Press, Cambridge, UK, 1989.

[10] A. P. Black, "Electrokinetic characteristics of hydrous oxides of aluminum and iron," in Principles and Applications of Water Chemistry: Proceedings of the Fourth Rudolfs Conference, Rutgers, S. D. Faust and J. V. Hunter, Eds., pp. 274-296, John Wiley \& Sons, New York, NY, USA, 1967.

[11] V. K. La Mer, "Coagulation symposium introduction," Journal of Colloid Science, vol. 19, no. 4, pp. 291-293, 1964.

[12] Y. Adachi, "Dynamic aspects of coagulation and flocculation," Advances in Colloid and Interface Science, vol. 56, pp. 1-31, 1995.

[13] G. Durand-Piana, F. Lafuma, and R. Audebert, "Flocculation and adsorption properties of cationic polyelectrolytes toward Na-montmorillonite dilute suspensions," Journal of Colloid And Interface Science, vol. 119, no. 2, pp. 474-480, 1987.

[14] J. Gregory, "Rates of flocculation of latex particles by cationic polymers," Journal of Colloid and Interface Science, vol. 42, no. 2, pp. 448-456, 1973.

[15] Y. Zhou, H. Yu, E. J. Wanless, G. J. Jameson, and G. V. Franks, "Influence of polymer charge on the shear yield stress of silica aggregated with adsorbed cationic polymers," Journal of Colloid and Interface Science, vol. 336, no. 2, pp. 533-543, 2009.

[16] W. C. K. Poon, "The physics of a model colloid-polymer mixture," Journal of Physics Condensed Matter, vol. 14, no. 33, pp. R859-R880, 2002.
[17] A. A. Potanin, R. De Rooij, D. Van den Ende, and J. Mellema, "Microrheological modeling of weakly aggregated dispersions," The Journal of Chemical Physics, vol. 102, no. 14, pp. 5845-5853, 1995.

[18] A. A. Potanin and W. B. Russel, "Fractal model of consolidation of weakly aggregated colloidal dispersions," Physical Review E: Statistical Physics, Plasmas, Fluids, and Related Interdisciplinary Topics, vol. 53, no. 4, pp. 3702-3709, 1996.

[19] E. M. Furst and J. P. Pantina, "Yielding in colloidal gels due to nonlinear microstructure bending mechanics," Physical Review E-Statistical, Nonlinear, and Soft Matter Physics, vol. 75, no. 5, Article ID 050402, 2007.

[20] J. E. Martin, J. P. Wilcoxon, and D. Adolf, "Critical exponents for the sol-gel transition," Physical Review A, vol. 36, no. 4, pp. 1803-1810, 1987.

[21] W. C. K. Poon and M. D. Haw, "Mesoscopic structure formation in colloidal aggregation and gelation," Advances in Colloid and Interface Science, vol. 73, pp. 71-126, 1997.

[22] M. Kolb, R. Botet, and R. Jullien, "Scaling of kinetically growing clusters," Physical Review Letters, vol. 51, no. 13, pp. 1123-1126, 1983.

[23] M. Kolb and H. J. Herrmann, "The sol-gel transition modelled by irreversible aggregation of clusters," Journal of Physics A: Mathematical and General, vol. 18, no. 8, pp. L435-L441, 1985.

[24] D. I. Verrelli, Drinking water treatment sludge production and dewaterability [Ph.D. thesis], The University of Melbourne, Melbourne, Australia, 2008, http://hdl.handle.net/11343/35087.

[25] J. N. Wilking, T. E. Angelini, A. Seminara, M. P. Brenner, and D. A. Weitz, "Biofilms as complex fluids," MRS Bulletin, vol. 36, no. 5, pp. 385-391, 2011.

[26] C. Wagner, P. Steffen, and S. Svetina, "Aggregation of red blood cells: from rouleaux to clot formation," Comptes Rendus Physique, vol. 14, no. 6, pp. 459-469, 2013.

[27] H. Bäumler, B. Neu, E. Donath, and H. Kiesewetter, "Basic phenomena of red blood cell rouleaux formation," Biorheology, vol. 36, no. 5-6, pp. 439-442, 1999.

[28] D. I. Verrelli, N. Haque, G. J. Dumsday et al., "Using life cycle assessment to guide process development for algal oil production," Internal Report, CSIRO, 2015.

[29] I. S. Aranson, "Collective behavior in out-of-equilibrium colloidal suspensions," Comptes Rendus Physique, vol. 14, no. 6, pp. 518-527, 2013.

[30] M. Hermansson, "The DLVO theory in microbial adhesion," Colloids and Surfaces B: Biointerfaces, vol. 14, no. 1-4, pp. 105119, 1999.

[31] A. T. Poortinga, R. Bos, W. Norde, and H. J. Busscher, "Electric double layer interactions in bacterial adhesion to surfaces," Surface Science Reports, vol. 47, no. 1, pp. 1-32, 2002.

[32] J. Schwarz-Linek, J. Arlt, A. Jepson et al., "Escherichia coli as a model active colloid: a practical introduction," Colloids and Surfaces B: Biointerfaces, vol. 137, pp. 2-16, 2016.

[33] J. Gregory, "Polymer adsorption and flocculation in sheared suspensions," Colloids and Surfaces, vol. 31, pp. 231-253, 1988.

[34] A. S. Michaels and J. C. Bolger, "Settling rates and sediment volumes of flocculated kaolin suspensions," Industrial \& Engineering Chemistry Fundamentals, vol. 1, no. 1, pp. 24-33, 1962.

[35] P. T. Spicer, S. E. Pratsinis, J. Raper, R. Amal, G. Bushell, and G. Meesters, "Effect of shear schedule on particle size, density, and structure during flocculation in stirred tanks," Powder Technology, vol. 97, no. 1, pp. 26-34, 1998. 
[36] C. B. Crawford, "Cohesion in an undisturbed sensitive clay," Géotechnique, vol. 13, no. 2, pp. 132-144, 1963.

[37] P. Meakin, "Formation of fractal clusters and networks by irreversible diffusion-limited aggregation," Physical Review Letters, vol. 51, no. 13, pp. 1119-1122, 1983.

[38] Z. Shao, A. S. Negi, and C. O. Osuji, "Role of interparticle attraction in the yielding response of microgel suspensions," Soft Matter, vol. 9, no. 22, pp. 5492-5500, 2013.

[39] C. R. I. Clayton, M. C. Matthews, and N. E. Simons, Site Investigation [A Handbook for Engineers], 2nd edition, 2004.

[40] P. Varadan and M. J. Solomon, "Direct visualization of flowinduced microstructure in dense colloidal gels by confocal laser scanning microscopy," Journal of Rheology, vol. 47, no. 4, pp. 943-968, 2003.

[41] R. G. Larson, The Structure and Rheology of Complex Fluids, Oxford University Press, New York, NY, USA, 1999.

[42] P. Coussot, Rheometry of Pastes, Suspensions, and Granular Materials. Applications in Industry and Environment, WileyInterscience, John Wiley \& Sons, Hoboken, NJ, USA, 2005.

[43] R. G. de Kretser, P. J. Scales, and D. V. Boger, "Compressive rheology : an overview," in Rheology Reviews 2003, D. M. Binding and K. Walters, Eds., pp. 125-165, British Society of Rheology, Abertswyth, Wales, 2003.

[44] M. Cloitre, R. Borrega, and L. Leibler, "Rheological aging and rejuvenation in microgel pastes," Physical Review Letters, vol. 85, no. 22, pp. 4819-4822, 2000.

[45] W. B. Russel and M. C. Grant, "Distinguishing between dynamic yielding and wall slip in a weakly flocculated colloidal dispersion," Colloids and Surfaces A: Physicochemical and Engineering Aspects, vol. 161, no. 2, pp. 271-282, 2000.

[46] G. Ovarlez, F. Mahaut, F. Bertrand, and X. Chateau, "Flows and heterogeneities with a vane tool: magnetic resonance imaging measurements," Journal of Rheology, vol. 55, no. 2, pp. 197-223, 2011.

[47] P. Saramito, "A new constitutive equation for elastoviscoplastic fluid flows," Journal of Non-Newtonian Fluid Mechanics, vol. 145, no. 1, pp. 1-14, 2007.

[48] E. C. Bingham, "The plasticity of solids," in Fluidity and Plasticity, chapter 8, pp. 215-240, McGraw-Hill, New York, NY, USA, 1922.

[49] H. A. Barnes, "The yield stress-a review or ' $\pi \alpha \nu \tau \alpha \rho \varepsilon$ 'everything flows?" Journal of Non-Newtonian Fluid Mechanics, vol. 81, no. 1-2, pp. 133-178, 1999.

[50] H. A. Barnes, "The 'Yield stress myth?' paper-21 years on," Applied Rheology, vol. 17, no. 4, pp. 43110-1-43110-5, 2007.

[51] H. A. Barnes and K. Walters, “The yield stress myth?" Rheologica Acta, vol. 24, no. 4, pp. 323-326, 1985.

[52] G. W. Scott Blair, "On the nature of 'yield-value,' Physics, vol. 4, no. 3, pp. 113-118, 1933.

[53] Q. D. Nguyen and D. V. Boger, "Measuring the flow properties of yield stress fluids," Annual Review of Fluid Mechanics, vol. 24, no. 1, pp. 47-88, 1992.

[54] R. T. Bonnecaze and J. F. Brady, "Yield stresses in electrorheological fluids," Journal of Rheology, vol. 36, no. 1, pp. 73-115, 1992.

[55] R. Houwink, Elasticity, Plasticity and Structure of Matter, Cambridge University Press, Cambridge, UK, 1937.

[56] A. E. James, D. J. A. Williams, and P. R. Williams, "Direct measurement of static yield properties of cohesive suspensions," Rheologica Acta, vol. 26, no. 5, pp. 437-446, 1987.
[57] P. Coussot, "Yield stress fluid flows: a review of experimental data," Journal of Non-Newtonian Fluid Mechanics, vol. 211, pp. 31-49, 2014.

[58] K. A. Dawson, "The glass paradigm for colloidal glasses, gels, and other arrested states driven by attractive interactions," Current Opinion in Colloid \& Interface Science, vol. 7, no. 3-4, pp. 218-227, 2002.

[59] K. N. Pham, G. Petekidis, D. Vlassopoulos, S. U. Egelhaaf, W. C. K. Poon, and P. N. Pusey, "Yielding behavior of repulsion- and attraction-dominated colloidal glasses," Journal of Rheology, vol. 52, no. 2, pp. 649-676, 2008.

[60] N. Koumakis and G. Petekidis, “Two step yielding in attractive colloids: transition from gels to attractive glasses," Soft Matter, vol. 7, no. 6, pp. 2456-2470, 2011.

[61] K. N. Pham, G. Petekidis, D. Vlassopoulos, S. U. Egelhaaf, P. N. Pusey, and W. C. K. Poon, "Yielding of colloidal glasses," Europhysics Letters, vol. 75, no. 4, pp. 624-630, 2006.

[62] D. I. Verrelli, D. R. Dixon, and P. J. Scales, "Effect of coagulation conditions on the dewatering properties of sludges produced in drinking water treatment," Colloids and Surfaces A, vol. 348, no. 1-3, pp. 14-23, 2009.

[63] P. Saramito, "A new elastoviscoplastic model based on the Herschel-Bulkley viscoplastic model," Journal of NonNewtonian Fluid Mechanics, vol. 158, no. 1-3, pp. 154-161, 2009.

[64] H. A. Barnes, J. F. Hutton, and K. Walters, An Introduction to Rheology, Elsevier Science, Elsevier, Amsterdam, The Netherlands, 1989.

[65] H. Markovitz, "Nonlinear steady-flow behavior," in Rheology Theory and Applications, F. R. Eirich, Ed., vol. 4, pp. 347-410, Academic Press, New York, NY, USA, 1967.

[66] B. M. Erwin, M. Cloitre, M. Gauthier, and D. Vlassopoulos, "Dynamics and rheology of colloidal star polymers," Soft Matter, vol. 6, no. 12, pp. 2825-2833, 2010.

[67] B. M. Erwin, D. Vlassopoulos, and M. Cloitre, "Rheological fingerprinting of an aging soft colloidal glass," Journal of Rheology, vol. 54, no. 4, pp. 915-939, 2010.

[68] L. Bergström, "Sedimentation of flocculated alumina suspensions: $\gamma$-ray measurements and comparison with model predictions," Journal of the Chemical Society, Faraday Transactions, vol. 88, no. 21, pp. 3201-3211, 1992.

[69] F. M. Auzerais, R. Jackson, and W. B. Russel, "The resolution of shocks and the effects of compressible sediments in transient settling," Journal of Fluid Mechanics, vol. 195, pp. 437-462, 1988.

[70] C. Shen, W. B. Russel, and F. M. Auzerais, "Colloidal gel filtration: experiment and theory," AIChE Journal, vol. 40, no. 11, pp. 1876-1891, 1994.

[71] R. M. L. Evans and L. Starrs, "Emergence of a stress transmission length-scale in transient gels," Journal of Physics: Condensed Matter, vol. 14, no. 10, pp. 2507-2529, 2002.

[72] P. Coussot, "Rheophysics of pastes: a review of microscopic modelling approaches," Soft Matter, vol. 3, no. 5, pp. 528-540, 2007.

[73] H. A. Barnes and Q. D. Nguyen, "Rotating vane rheometry-a review," Journal of Non-Newtonian Fluid Mechanics, vol. 98, no. 1, pp. 1-14, 2001.

[74] N. Pashias, D. V. Boger, J. Summers, and D. J. Glenister, "A fifty cent rheometer for yield stress measurement," Journal of Rheology, vol. 40, no. 6, pp. 1179-1189, 1996.

[75] N. Pashias, D. V. Boger, K. J. Summers, and D. J. Glenister, "A fifty cent rheometer for waste management of environmentally 
sensitive ore tailings," Mineral Processing and Extractive Metallurgy Review, vol. 20, no. 1, pp. 115-122, 1999.

[76] N. E. McTigue, D. A. Murray, and M. Wang, "Landfill options," in Management of Water Treatment Plant Residuals (Technology Transfer Handbook), p. 132, US Environmental Protection Agency, American Society of Civil Engineers, American Water Works Association, Cincinnati, Ohio, USA, 1996.

[77] D. A. Cornwell, Water Treatment Residuals Engineering, AWWA Research Foundation, American Water Works Association, Denver, Colo, USA, 2006.

[78] A. W. Saak, H. M. Jennings, and S. P. Shah, "A generalized approach for the determination of yield stress by slump and slump flow," Cement and Concrete Research, vol. 34, no. 3, pp. 363-371, 2004.

[79] D. G. Bika, M. Gentzler, and J. N. Michaels, "Mechanical properties of agglomerates," Powder Technology, vol. 117, no. 12, pp. 98-112, 2001.

[80] J. D. Sherwood, G. H. Meeten, C. A. Farrow, and N. J. Alderman, "Squeeze-film rheometry of non-uniform mudcakes," Journal of Non-Newtonian Fluid Mechanics, vol. 39, no. 3, pp. 311-334, 1991.

[81] P. H. T. Uhlherr, T. Fang, and Z. Zhou, "Determination of yield stress from initiation of motion on an inclined plane," Mineral Processing and Extractive Metallurgy Review, vol. 20, no. 1, pp. 93-114, 1999.

[82] P. Coussot and S. Boyer, "Determination of yield stress fluid behaviour from inclined plane test," Rheologica Acta, vol. 34, no. 6, pp. 534-543, 1995.

[83] Z. D. Jastrzebski, "Entrance effects and wall effects in an extrusion rheometer during the flow of concentrated suspensions," Industrial and Engineering Chemistry Fundamentals, vol. 6, no. 3, pp. 445-454, 1967.

[84] N. Tetlow, A. L. Graham, M. S. Ingber, S. R. Subia, L. A. Mondy, and S. A. Altobelli, "Particle migration in a Couette apparatus: experiment and modeling," Journal of Rheology, vol. 42, no. 2, pp. $307-327,1998$.

[85] A. S. Yoshimura, R. K. Prud'homme, H. M. Princen, and A. D. Kiss, "A comparison of techniques for measuring yield stresses," Journal of Rheology, vol. 31, no. 8, pp. 699-710, 1987.

[86] K. Hyun, M. Wilhelm, C. O. Klein et al., "A review of nonlinear oscillatory shear tests: analysis and application of large amplitude oscillatory shear (LAOS)," Progress in Polymer Science, vol. 36, no. 12, pp. 1697-1753, 2011.

[87] P. Coussot, Q. D. Nguyen, H. T. Huynh, and D. Bonn, "Avalanche behavior in yield stress fluids," Physical Review Letters, vol. 88, no. 17, pp. 175501-1-175501-4, 2002.

[88] K. Terzaghi, R. B. Peck, and G. Mesri, Soil Mechanics in Engineering Practice, Wiley-Interscience, John Wiley \& Sons, New York, NY, USA, 3rd edition, 1996.

[89] Standards Australia, "Methods of testing soils for engineering purposes. Method 6.2.1: soil strength and consolidation testsdetermination of the shear strength of a soil-field test using a vane," AS 1289.6.2.1-2001, Standards Australia, 2001.

[90] Q. D. Nguyen and D. V. Boger, "Yield stress measurement for concentrated suspensions," Journal of Rheology, vol. 27, no. 4, pp. 321-349, 1983.

[91] D. Doraiswamy, A. Mujumdar, I. Tsao, A. Beris, S. Danforth, and A. Metzner, "The Cox-Merz rule extended: a rheological model for concentrated suspensions and other materials with a yield stress," Journal of Rheology, vol. 35, no. 4, pp. 647-685, 1991.
[92] I. Cheddadi, P. Saramito, and F. Graner, "Steady Couette flows of elastoviscoplastic fluids are nonunique," Journal of Rheology, vol. 56, no. 1, pp. 213-239, 2012.

[93] D. M. Wood, Soil Behaviour and Critical State Soil Mechanics, Cambridge University, Cambridge, UK, 1990.

[94] G. A. Leonards, "Engineering properties of soils," in Foundation Engineering, G. A. Leonards, Ed., pp. 66-240, McGraw-Hill, New York, NY, USA, 1962.

[95] J. Foong, Yielding, linear and non-linear viscoelastic behaviour of concentrated coagulated suspensions [Ph.D. thesis], The University of Melbourne, Melbourne, Australia, 2008.

[96] E. D. Shchukin and P. A. Rehbinder, "Mechanism of the elastic lag of low-concentration structured suspensions of bentonite," Kolloidn Zhurnal, vol. 33, pp. 373-379, 1971.

[97] W. Świdziński, "Methods of determination of elastic moduli of particulate materiais-brief review," Archives of HydroEngineering and Environmental Mechanics, vol. 47, no. 1-4, pp. 27-50, 2000.

[98] V. Gopalakrishnan and C. F. Zukoski, "Effect of attractions on shear thickening in dense suspensions," Journal of Rheology, vol. 48, no. 6, pp. 1321-1344, 2004.

[99] A. D. Stickland, A. Kumar, T. E. Kusuma et al., "The effect of premature wall yield on creep testing of strongly flocculated suspensions," Rheologica Acta, vol. 54, no. 5, pp. 337-352, 2015.

[100] H. A. Barnes, "A review of the slip (wall depletion) of polymer solutions, emulsions and particle suspensions in viscometers: its cause, character, and cure," Journal of Non-Newtonian Fluid Mechanics, vol. 56, no. 3, pp. 221-251, 1995.

[101] P. Ballesta, G. Petekidis, L. Isa, W. C. K. Poon, and R. Besseling, "Wall slip and flow of concentrated hard-sphere colloidal suspensions," Journal of Rheology, vol. 56, no. 5, pp. 1005-1037, 2012.

[102] P. Ballesta, N. Koumakis, R. Besseling, W. C. K. Poon, and G. Petekidis, "Slip of gels in colloid-polymer mixtures under shear," Soft Matter, vol. 9, no. 12, pp. 3237-3245, 2013.

[103] R. T. Bonnecaze and M. Cloitre, "Micromechanics of soft particle glasses," in High Solid Dispersions, M. Cloitre, Ed., vol. 236, chapter 90, pp. 117-161, Springer, Berlin, Germany, 2010.

[104] A. N. Gent, J. B. Suh, and S. G. Kelly III, "Mechanics of rubber shear springs," International Journal of Non-Linear Mechanics, vol. 42, no. 2, pp. 241-249, 2007.

[105] A. Sierou and J. F. Brady, "Rheology and microstructure in concentrated noncolloidal suspensions," Journal of Rheology, vol. 46, no. 5, pp. 1031-1056, 2002.

[106] O. Reynolds, "LVII. On the dilatancy of media composed of rigid particles in contact. With experimental illustrations," Philosophical Magazine Series 5, vol. 20, no. 127, pp. 469-481, 1885.

[107] M. S. Green and A. V. Tobolsky, "A new approach to the theory of relaxing polymeric media," The Journal of Chemical Physics, vol. 14, no. 2, pp. 80-92, 1946.

[108] R. K. Gupta and S. N. Bhattacharya, "Effect of mixing on shear rheology of EVA nanocomposites," Korea-Australia Rheology Journal, vol. 22, no. 3, pp. 197-203, 2010.

[109] A. J. M. Spencer, Continuum Mechanics, Longman, London, UK, 1980.

[110] V. M. Segal, "Severe plastic deformation: simple shear versus pure shear," Materials Science and Engineering A, vol. 338, no. 1-2, pp. 331-344, 2002.

[111] N. S. Ottosen and M. Ristinmaa, The Mechanics of Constitutive Modeling, Elsevier Science, Amsterdam, The Netherlands, 2005. 
[112] M. C. Matthews, "Engineering application of direct and simple shear testing," Ground Engineering, vol. 21, no. 2, pp. 613-621, 1988.

[113] H.-C. Wu, Continuum Mechanics and Plasticity, Chapman \& Hall/CRC, Boca Raton, Fla, USA, 2005.

[114] R. S. Rivlin, "Large elastic deformations of isotropic materials. IV. Further developments of the general theory," Philosophical Transactions of the Royal Society of London Series A: Mathematical and Physical Sciences, vol. 241, no. 835, pp. 379-397, 1948.

[115] C. W. Macosko, Rheology: Principles, Measurements, and Applications, VCH, New York, NY, USA, 1994.

[116] T. E. Hardingham, H. Muir, M. K. Kwan, W. M. Lai, and V. C. Mow, "Viscoelastic properties of proteoglycan solutions with varying proportions present as aggregates," Journal of Orthopaedic Research, vol. 5, no. 1, pp. 36-46, 1987.

[117] A. Morley, Strength of Materials, Longmans, Green \& Company, London, UK, 11th edition, 1954.

[118] J. B. Suh, A. N. Gent, and S. G. Kelly III, "Shear of rubber tube springs," International Journal of Non-Linear Mechanics, vol. 42, no. 9, pp. 1116-1126, 2007.

[119] R. B. Bird, R. C. Armstrong, and O. Hassager, "Codeformational models," in Dynamics of Polymeric Liquids Volume 1: Fluid Mechanics, pp. 417-461, John Wiley \& Sons, New York, NY, USA, 1977.

[120] A. S. Negi and C. O. Osuji, "New insights on fumed colloidal rheology-shear thickening and vorticity-aligned structures in flocculating dispersions," Rheologica Acta, vol. 48, no. 8, pp. 871881, 2009.

[121] J. M. Broadbent and A. S. Lodge, "Determination of normalstress differences in steady shear flow: III. A wide-gap concentric cylinder apparatus," Rheologica Acta, vol. 10, pp. 557-573, 1971.

[122] J. J. Windheuser, J. Misra, S. P. Eriksen, and T. Higuchi, "Physics of tablet compression XIII. Development of die-wall pressure during compression of various materials," Journal of Pharmaceutical Sciences, vol. 52, pp. 767-772, 1963.

[123] H. M. Laun, "Normal stresses in extremely shear thickening polymer dispersions," Journal of Non-Newtonian Fluid Mechanics, vol. 54, pp. 87-108, 1994.

[124] G. M. Channell, "Effects of normal stresses on the shear yield stress," in Mechanics of Aggregated Alumina Suspensions: Behavior under Shear and Compression, pp. 111-132, Chemical Engineering, University of Illinois at Urbana-Champaign, Champaign, Ill, USA, 1999.

[125] A. D. Stickland and P. J. Scales, "The effect of compressional load on shear yield stress," in Proceedings of the 6th KoreanAustralian Rheology Conference (KARC '11), p. 9, Korean Society of Rheology, Daejeon, Republic of Korea, September 2011.

[126] R. F. Scott, Principles of Soil Mechanics, Addison-Wesley, Reading, Mass, USA, 1963.

[127] G. Ovarlez, Q. Barral, and P. Coussot, "Three-dimensional jamming and flows of soft glassy materials," Nature Materials, vol. 9, no. 2, pp. 115-119, 2010.

[128] A. M. Freudenthal and P. F. Gou, "Second order effects in the theory of plasticity," Acta Mechanica, vol. 8, no. 1-2, pp. 34-52, 1969.

[129] D. Su, Z.-L. Wang, and F. Xing, "A two-parameter expression for failure surfaces," Computers and Geotechnics, vol. 36, no. 3, pp. 517-524, 2009.

[130] M.-H. Yu, "Advances in strength theories for materials under complex stress state in the 20th century," Applied Mechanics Reviews, vol. 55, no. 3, pp. 169-218, 2002.
[131] J. M. Brader, T. Voigtmann, M. Fuchs, R. G. Larson, and M. E. Cates, "Glass rheology: from mode-coupling theory to a dynamical yield criterion," Proceedings of the National Academy of Sciences of the United States of America, vol. 106, no. 36, pp. 15186-15191, 2009.

[132] T. F. F. Farage and J. M. Brader, "Three-dimensional flow of colloidal glasses," Journal of Rheology, vol. 56, no. 2, pp. 259278, 2012.

[133] A. Arsac, C. Carrot, J. Guillet, and P. Revenu, "Problems originating from the use of the Gordon-Schowalter derivative in the Johnson-Segalman and related models in various shear flow situations," Journal of Non-Newtonian Fluid Mechanics, vol. 55, no. 1, pp. 21-36, 1994.

[134] L. Chang, K. Friedrich, A. K. Schlarb, R. Tanner, and L. Ye, "Shear-thickening behaviour of concentrated polymer dispersions under steady and oscillatory shear," Journal of Materials Science, vol. 46, no. 2, pp. 339-346, 2011.

[135] P. Coussot, A. Ragouilliaux, G. Ovarlez, and B. Herzhaft, "Transition from a simple yield stress fluid to a thixotropic material," in Proceedings of the 15th International Congress on Rheology, A. Co, L. G. Leal, R. H. Colby, and A. J. Giacomin, Eds., pp. 713-715, American Institute of Physics, 2008.

[136] P. Coussot, J. S. Raynaud, F. Bertrand et al., "Coexistence of liquid and solid phases in flowing soft-glassy materials," Physical Review Letters, vol. 88, no. 21, Article ID 218301, 2002.

[137] B. Kaffashi, M. Barmar, and J. Eyvani, "The steady state and dynamic rheological properties of telechelic associative polymer solutions," Colloids and Surfaces A: Physicochemical and Engineering Aspects, vol. 254, no. 1-3, pp. 125-130, 2005.

[138] A. I. Jomha and P. A. Reynolds, "An experimental study of the first normal stress difference-shear stress relationship in simple shear flow for concentrated shear thickening suspensions," Rheologica Acta, vol. 32, no. 5, pp. 457-464, 1993.

[139] Y. Yurkovetsky and J. F. Morris, "Particle pressure in sheared Brownian suspensions," Journal of Rheology, vol. 52, no. 1, pp. 141-164, 2008.

[140] Y. Otsubo, "Normal stress behavior of highly elastic suspensions," Journal of Colloid and Interface Science, vol. 163, no. 2, pp. 507-511, 1994.

[141] J. Katona, V. Sovilj, and L. Petrovic, "Influence of sodium dodecylsulfate and hydroxypropylmethyl cellulose interaction on visco-elastic properties of the solution," Annals of West University of Timisoara Series of Chemistry, vol. 16, pp. 53-60, 2007.

[142] S. Lin-Gibson, J. A. Pathak, E. A. Grulke, H. Wang, and E. K. Hobbie, "Elastic flow instability in nanotube suspensions," Physical Review Letters, vol. 92, no. 4, Article ID 048302, 2004.

[143] C. O. Osuji and D. A. Weitz, "Highly anisotropic vorticity aligned structures in a shear thickening attractive colloidal system," Soft Matter, vol. 4, no. 7, pp. 1388-1392, 2008.

[144] R. de Rooij, D. van den Ende, M. H. G. Duits, and J. Mellema, "Elasticity of weakly aggregating polystyrene latex dispersions," Physical Review E, vol. 49, no. 4, pp. 3038-3049, 1994.

[145] H. M. Wyss, E. V. Tervoort, and L. J. Gauckler, "Mechanics and microstructures of concentrated particle gels," Journal of the American Ceramic Society, vol. 88, no. 9, pp. 2337-2348, 2005.

[146] H. Leaderman, R. G. Smith, and R. W. Jones, "Rheology of polyisobutylene. II. Low molecular weight polymers," Journal of Polymer Science, vol. 14, no. 73, pp. 47-80, 1954.

[147] B. D. Coleman and H. Markovitz, "Normal stress effects in second-order fluids," Journal of Applied Physics, vol. 35, no. 1, pp. 1-9, 1964. 
[148] H. M. Laun, "Prediction of elastic strains of polymer melts in shear and elongation," Journal of Rheology, vol. 30, no. 3, pp. 459-501, 1986.

[149] B. M. Erwin, S. A. Rogers, M. Cloitre, and D. Vlassopoulos, "Examining the validity of strain-rate frequency superposition when measuring the linear viscoelastic properties of soft materials," Journal of Rheology, vol. 54, no. 2, pp. 187-195, 2010.

[150] J. Feda, Creep of Soils and Related Phenomena, Elsevier, Amsterdam, The Netherlands, 1992.

[151] M. A. Yukselen and J. Gregory, "The reversibility of floc breakage," International Journal of Mineral Processing, vol. 73, no. 2-4, pp. 251-259, 2004.

[152] A. S. Lodge, "A network theory of flow birefringence and stress in concentrated polymer solutions," Transactions of the Faraday Society, vol. 52, pp. 120-130, 1956.

[153] N. Adams and A. S. Lodge, "Rheological properties of concentrated polymer solutions II. A cone-and-plate and parallel-plate pressure distribution apparatus for determining normal stress differences in steady shear flow," Philosophical Transactions of the Royal Society of London Series A: Mathematical and Physical Sciences, vol. 256, no. 1068, pp. 149-184, 1964.

[154] Y. H. Wen, H. C. Lin, C. H. Li, and C. C. Hua, "An experimental appraisal of the Cox-Merz rule and Laun's rule based on bidisperse entangled polystyrene solutions," Polymer, vol. 45, no. 25, pp. 8551-8559, 2004.

[155] C. Holmqvist and A. Dahlkild, "Consolidation of sheared, strongly flocculated suspensions," AIChE Journal, vol. 54, no. 4, pp. 924-939, 2008.

[156] L. Isa, R. Besseling, A. B. Schofield, and W. C. K. Poon, "Quantitative imaging of concentrated suspensions under flow," in High Solid Dispersions, M. Cloitre, Ed., vol. 236 of Advances in Polymer Science, chapter 38, pp. 163-202, Springer, Berlin, Germany, 2010.

[157] D. J. Klingenberg and C. F. Zukoski IV, "Studies on the steadyshear behavior of electrorheological suspensions," Langmuir, vol. 6, no. 1, pp. 15-24, 1990.

[158] J. C. Maxwell, "On elasticity and viscosity," in Theory of Heat, chapter 21, pp. 274-281, Longmans, Green \& Company, London, UK, 3rd edition, 1872 .

[159] R. Hill, The Mathematical Theory of Plasticity, Oxford University Press, Oxford, UK, 1950.

[160] B. J. Park, F. F. Fang, and H. J. Choi, "Magnetorheology: materials and application," Soft Matter, vol. 6, no. 21, pp. 52465253, 2010.

[161] E. Brown, N. A. Forman, C. S. Orellana et al., "Generality of shear thickening in dense suspensions," Nature Materials, vol. 9, no. 3, pp. 220-224, 2010.

[162] H. J. Choi and M. S. Jhon, "Electrorheology of polymers and nanocomposites," Soft Matter, vol. 5, no. 8, pp. 1562-1567, 2009.

[163] I. M. Krieger, "Correspondence: comments on a manuscript by Doraiswamy et al," Journal of Rheology, vol. 36, no. 1, pp. 215216, 1992. 

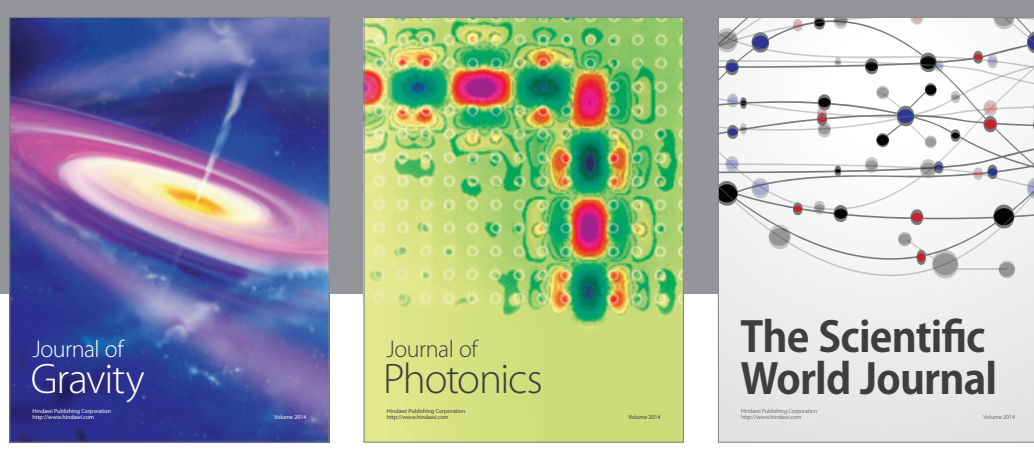

The Scientific World Journal
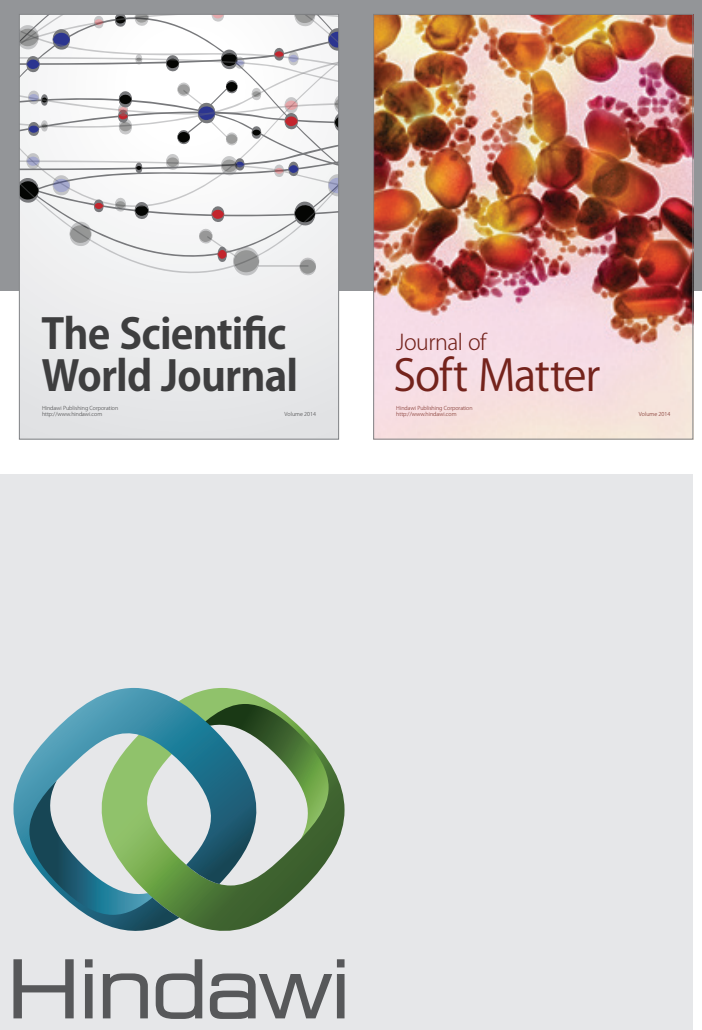

Submit your manuscripts at

http://www.hindawi.com

nternational Journal of

Statistical Mechanics
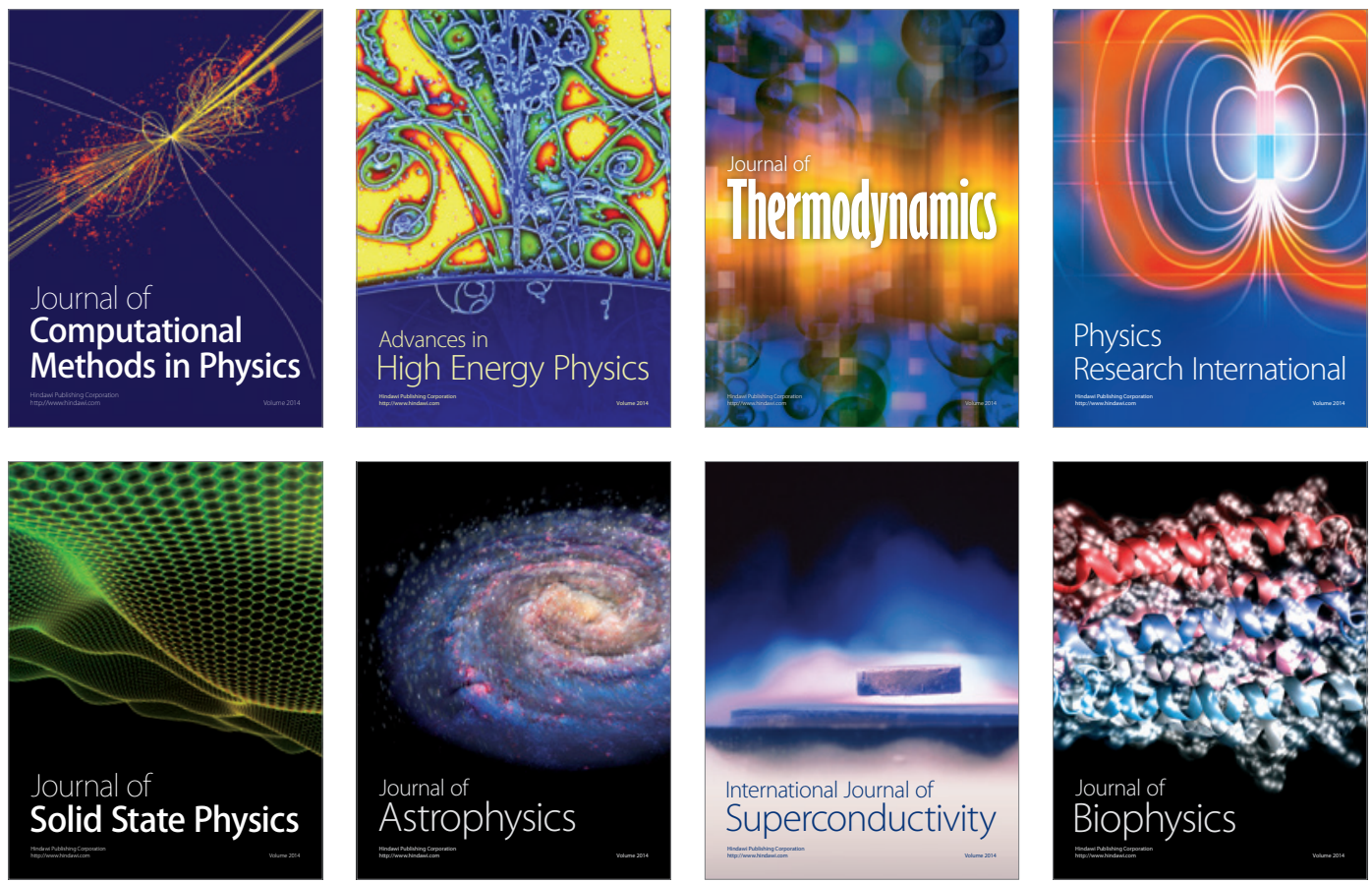
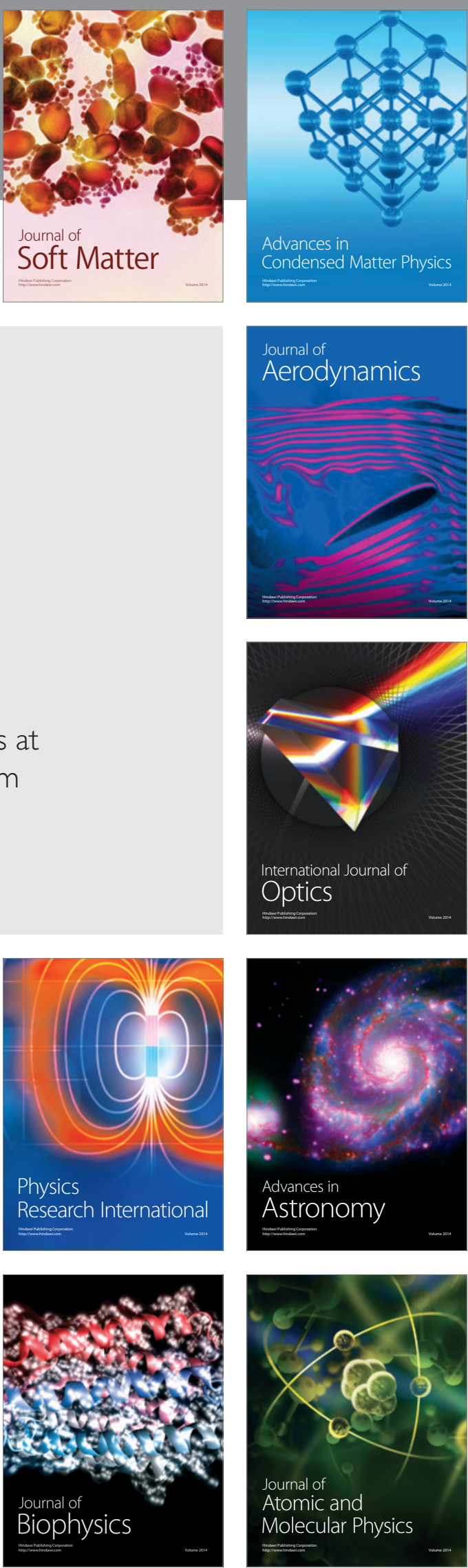